\title{
PHYSICAL CHARACTERISTICS OF G331.5-0.1: THE LUMINOUS CENTRAL REGION OF A GIANT MOLECULAR CLOUD
}

\author{
Manuel Merello $^{1,2}$, Leonardo Bronfman $^{1}$, Guido Garay ${ }^{1}$, Lars-Åke Nyman $^{3}$, \\ Neal J. Evans II ${ }^{2}$, and C. Malcolm Walmsley ${ }^{4,5}$ \\ ${ }^{1}$ Departamento de Astronomía, Universidad de Chile, Casilla 36-D, Santiago, Chile \\ ${ }^{2}$ University of Texas at Austin, 1 University Station, Austin, TX 78712, USA \\ ${ }^{3}$ Joint ALMA Observatory (JAO), Alonso de Cordova 3107, Vitacura, Santiago, Chile \\ ${ }^{4}$ Osservatorio Astrofisico di Arcetri, Largo E. Fermi 5, I-50125 Firenze, Italy \\ 5 Dublin Institute of Advanced Studies, Fitzwilliam Place 31, Dublin 2, Ireland \\ Received 2012 December 7; accepted 2013 May 26; published 2013 August 14
}

\begin{abstract}
We report molecular line and dust continuum observations toward the high-mass star-forming region G331.5-0.1, one of the most luminous regions of massive star formation in the Milky Way, located at the tangent region of the Norma spiral arm, at a distance of $7.5 \mathrm{kpc}$. Molecular emission was mapped toward the G331.5-0.1 GMC in the $\mathrm{CO}(J=1 \rightarrow 0)$ and $\mathrm{C}^{18} \mathrm{O}(J=1 \rightarrow 0)$ lines with NANTEN, while its central region was mapped in $\mathrm{CS}(J=2 \rightarrow 1$ and $J=5 \rightarrow 4)$ with $\operatorname{SEST}$, and in $\operatorname{CS}(J=7 \rightarrow 6)$ and ${ }^{13} \mathrm{CO}(J=3 \rightarrow 2)$ with ASTE. Continuum emission mapped at $1.2 \mathrm{~mm}$ with SIMBA and at $0.87 \mathrm{~mm}$ with LABOCA reveal the presence of six compact and luminous dust clumps, making this source one of the most densely populated central regions of a GMC in the Galaxy. The dust clumps are associated with molecular gas and they have the following average properties: size of $1.6 \mathrm{pc}$, mass of $3.2 \times 10^{3} M_{\odot}$, molecular hydrogen density of $3.7 \times 10^{4} \mathrm{~cm}^{-3}$, dust temperature of $32 \mathrm{~K}$, and integrated luminosity of $5.7 \times 10^{5} L_{\odot}$, consistent with values found toward other massive star-forming dust clumps. The $\mathrm{CS}$ and ${ }^{13} \mathrm{CO}$ spectra show the presence of two velocity components: a high-velocity component at $\sim-89 \mathrm{~km} \mathrm{~s}^{-1}$, seen toward four of the clumps, and a low-velocity component at $\sim-101 \mathrm{~km} \mathrm{~s}^{-1}$ seen toward the other two clumps. Radio continuum emission is present toward four of the molecular clumps, with spectral index estimated for two of them of $0.8 \pm 0.2$ and $1.2 \pm 0.2$. A high-velocity molecular outflow is found at the center of the brightest clump, with a line width of $26 \mathrm{~km} \mathrm{~s}^{-1}$ (FWHM) in $\mathrm{CS}(J=7 \rightarrow 6)$. Observations of $\operatorname{SiO}(J=7 \rightarrow 6$ and $J=8 \rightarrow 7)$, and $\operatorname{SO}\left(J_{K}=8_{8} \rightarrow 7_{7}\right.$ and $\left.J_{K}=8_{7} \rightarrow 7_{6}\right)$ lines provide estimates of the gas rotational temperature toward this outflow $>120 \mathrm{~K}$ and $>75 \mathrm{~K}$, respectively.
\end{abstract}

Key words: ISM: clouds - ISM: molecules - stars: formation

Online-only material: color figures

\section{INTRODUCTION}

Massive stars play a key role in the evolution of the Galaxy; hence they are important objects of study in astrophysics. Although their number is much smaller than the low mass stars, they are the principal source of heavy elements and UV radiation, affecting the process of formation of stars and planets (Bally et al. 2005) and the physical, chemical, and morphological structure of galaxies (e.g., Kennicutt 1998, 2005).

Diverse studies show that massive stars are formed in the denser regions of giant molecular clouds (GMCs; Churchwell et al. 1990; Cesaroni et al. 1991; Plume et al. 1992; Bronfman et al. 1996). The formation process of high-mass stars is, however, still under debate (Garay \& Lizano 1999). Observationally, recently formed massive stars cannot be detected at optical wavelengths because they are embedded within regions with high dust extinction. Another problem arises from theoretical considerations: a star with a mass larger than eight solar masses starts burning hydrogen during the accretion phase and the radiation pressure may halt or even reverse the infall process (e.g., Larson \& Starrfield 1971; Kahn 1974; Yorke \& Kruegel 1977; Wolfire \& Cassinelli 1987; Edgar \& Clarke 2004; Kuiper et al. 2010). Due to these difficulties, a paradigm that explains the formation of massive stars does not exist (Zinnecker \& Yorke 2007; McKee \& Ostriker 2007), contrary to the successful model accepted for low mass stars. Understanding the physical process that dominates during the early stages of the formation of massive stars and its influence back on its harboring molecular gas requires a study of the physical conditions of the environment previous to the star formation or at very early stages.

The object of our study is one of the most massive GMCs in the Southern Galaxy, at $l=331.5$ and $b=-0.1$, in the tangent region of the Norma spiral arm (Bronfman et al. 1985, 1989). Several observations have shown that the central region of this GMC harbors one of the most extended and luminous regions of massive star formation in the Galactic disk. Single dish radio continuum observations show the presence of an extended region of ionized gas (G331.5-0.1), with an angular size of $\sim 3^{\prime}-4^{\prime}$ (projected size $6.5-8.7 \mathrm{pc}$ at a distance of $7.5 \mathrm{kpc}$ ), close to the peak CO position of the GMC (Shaver \& Goss 1970; Caswell \& Haynes 1987). From observations of the H109 $\alpha$ and $\mathrm{H} 110 \alpha$ hydrogen recombination lines, Caswell \& Haynes (1987) determined that the line central velocity of the ionized gas is $\sim-89 \mathrm{~km} \mathrm{~s}^{-1}$, similar to the peak velocity of the GMC. The dynamical timescale of the region of ionized gas is $\sim 8.5 \times 10^{5} \mathrm{yr}$, and the rate of ionizing photons required to excite the $\mathrm{H}$ II region is $1.1 \times 10^{50} \mathrm{~s}^{-1}$. In addition, IRAS observations show the presence of an extended source, with an angular size similar to that of the ionized region and a FIR luminosity of $3.6 \times 10^{6} L_{\odot}$.

While both the ionizing photon rate and the FIR luminosity could be provided by a single $\mathrm{O} 4$ zero-age main sequence (ZAMS) star, it is most likely that the ionization and the heating of the region is provided by a cluster of massive OB stars. This explanation is supported by Midcourse Space Experiment (MSX) and Spitzer Space Telescope-GLIMPSE survey data, which show that there are several sites of massive star formation 
Table 1

Parameters of the Molecular Line Observations

\begin{tabular}{|c|c|c|c|c|c|c|c|c|c|}
\hline Telescope & Line & $\begin{array}{c}\text { Frequency } \\
(\mathrm{MHz})\end{array}$ & $\begin{array}{c}\text { Beam } \\
\text { (FWHM) }\end{array}$ & $\eta_{\mathrm{mb}}$ & Pos. Obs. & Spacing & $\begin{array}{l}t_{\text {in }} \\
(\mathrm{s})\end{array}$ & $\begin{array}{c}\Delta v \\
\left(\mathrm{~km} \mathrm{~s}^{-1}\right)\end{array}$ & $\begin{array}{c}\text { Noise } \\
(\mathrm{K})\end{array}$ \\
\hline \multirow[t]{2}{*}{ NANTEN } & $\mathrm{CO}(1 \rightarrow 0)$ & 115271.202 & $2 ! 6$ & 0.89 & 585 & $2 ! 5$ & $\leqslant 60$ & 0.15 & 0.35 \\
\hline & $\mathrm{C}^{18} \mathrm{O}(1 \rightarrow 0)$ & 109782.173 & $2: 6$ & 0.89 & 440 & 2.5 & $\leqslant 600$ & 0.15 & 0.1 \\
\hline \multirow[t]{2}{*}{ SEST } & $\mathrm{CS}(2 \rightarrow 1)$ & 97980.968 & $52^{\prime \prime}$ & 0.73 & 81 & $45^{\prime \prime}$ & 180 & 0.130 & 0.06 \\
\hline & $\mathrm{CS}(5 \rightarrow 4)$ & 244935.644 & $22^{\prime \prime}$ & 0.48 & 81 & $45^{\prime \prime}$ & 180 & 0.052 & 0.08 \\
\hline \multirow[t]{2}{*}{ ASTE } & ${ }^{13} \mathrm{CO}(3 \rightarrow 2)$ & 330587.957 & $22^{\prime \prime}$ & 0.61 & 167 & $22^{\prime \prime} .5$ & 240 & 0.113 & 0.1 \\
\hline & $\mathrm{CS}(7 \rightarrow 6)$ & 342882.950 & $22^{\prime \prime}$ & 0.61 & 167 & $22^{\prime \prime} .5$ & 240 & 0.109 & 0.1 \\
\hline \multirow[t]{4}{*}{ APEX } & $\mathrm{SiO}(7 \rightarrow 6)$ & 303926.809 & $20^{\prime \prime}$ & 0.7 & 1 & $\cdots$ & 670 & 0.48 & 0.04 \\
\hline & $\mathrm{SiO}(8 \rightarrow 7)$ & 347330.635 & $17^{\prime \prime} .6$ & 0.7 & 1 & $\cdots$ & 670 & 0.42 & 0.04 \\
\hline & $\mathrm{SO}\left(8_{7} \rightarrow 7_{6}\right)$ & 304077.844 & $20^{\prime \prime}$ & 0.7 & 1 & $\cdots$ & 680 & 0.48 & 0.04 \\
\hline & $\mathrm{SO}\left(8_{8} \rightarrow 7_{7}\right)$ & 344310.612 & $17^{\prime \prime} .6$ & 0.7 & 1 & $\cdots$ & 680 & 0.48 & 0.05 \\
\hline
\end{tabular}

spread over a region of $\sim 6^{\prime}$ in diameter. Bronfman et al. (2000) showed that toward the $l=331^{\circ}$ galactic longitude, there is a peak in the number density of IRAS sources with colors of ultracompact $\mathrm{H}_{\mathrm{II}}$ (UCHII) regions and associated with $\mathrm{CS}(2 \rightarrow 1)$ emission, which are thought to correspond to massive star-forming regions.

Evidence of active star formation in the G331.5-0.1 GMC is further provided by the detection of $\mathrm{OH}$ and methanol maser emission (Goss et al. 1970; Caswell et al. 1980; Caswell 1997, 1998; Pestalozzi et al. 2005), as well as $\mathrm{H}_{2} \mathrm{O}$ maser emission (J. L. Caswell 2013, private communication). Hydroxyl masers are known to be excellent indicators of UCHII regions, whereas methanol maser emission is a common phenomenon in regions of massive star formation (Menten et al. 1986) and $\mathrm{H}_{2} \mathrm{O}$ maser emission is thought to be a probe of the earliest stages of massive star formation (Furuya 2003). Also, within a radius of 4.5 centered on the peak position of G331.5-0.1 there are three objects from the Red MSX Source (RMS) catalog of massive young stellar objects (MYSOs) candidates (Urquhart et al. 2008), further indicating that the G331.5-0.1 central region is an active region of massive star formation. In addition, Bronfman et al. (2008) showed the presence of a compact, extremely high velocity molecular outflow in the G331.5-0.1 region, suggesting that it is driven by one of the most luminous and massive objects presently known. The G331.5-0.1 region is thus an ideal region to study extremely active massive star formation in GMCs.

Here we present observations and a thorough analysis of the G331.5-0.1 region with dust continuum and molecular line emission data. Section 2 describes the telescopes used and methods of data acquisition. Section 3 describes the results of our study at several scales, starting from GMCs $(\sim 100 \mathrm{pc})$, to molecular clumps sizes $(\sim 1 \mathrm{pc})$ and reaching sub-parsec scales in our interferometric data. This analysis considers dust emission and different molecular line tracers, along with mid-infrared and far-infrared data from the Spitzer, MSX, and IRAS telescopes, and free-free emission at $\mathrm{cm}$ wavelengths. Section 4 describes the physical parameters derived from our observations, including mass, density, and bolometric luminosity estimates. We also include a rotational temperature analysis for a massive and energetic molecular outflow in the G331.5-0.1 GMC central region. Our results are summarized in Section 5.

\section{OBSERVATIONS}

We observed emission in several molecular lines and continuum bands using several telescopes located in northern Chile: the NANTEN telescope of Nagoya University at the Las
Table 2

Parameters of Continuum Observations

\begin{tabular}{lcccc}
\hline \hline Telescope & $\begin{array}{c}\text { Frequency } \\
(\mathrm{GHz})\end{array}$ & $\begin{array}{c}\text { Bandwidth } \\
(\mathrm{GHz})\end{array}$ & $\begin{array}{c}\text { Beam Width } \\
(\mathrm{FWHM})\end{array}$ & $\begin{array}{c}\text { Noise } \\
\left(\mathrm{mJy} \mathrm{beam}^{-1}\right)\end{array}$ \\
\hline SEST & 250 & 90 & $24^{\prime \prime}$ & 50 \\
\hline APEX & 345 & 60 & $18^{\prime \prime} .6$ & 50 \\
\hline ATCA & 4.8 & 0.128 & $2^{\prime \prime} .7 \times 1^{\prime \prime} .8$ & 0.41 \\
& 8.6 & 0.128 & $1^{\prime \prime} .5 \times 1^{\prime \prime} .0$ & 0.37 \\
\hline
\end{tabular}

Campanas observatory, the $15 \mathrm{~m}$ Swedish-ESO Submillimetre Telescope (SEST) at La Silla, the $10 \mathrm{~m}$ Atacama Submillimeter Telescope Experiment (ASTE) at Pampa la Bola, and the $12 \mathrm{~m}$ Atacama Pathfinder Experiment (APEX) located in Llano Chajnantor. We also made observations using the Australia Telescope Compact Array (ATCA) located at Narrabri, Australia. The basic parameters of the molecular line observations are summarized in Table 1. Column 1 gives the telescope used, Columns 2 and 3 give the observed transition and line frequency, respectively. Columns 4 and 5 give the half-power beam width and the main beam efficiency. Columns 6-10 give, respectively, the number of positions observed, the angular spacing, the integration time on source per position, the channel width, and the resulting rms noise in antenna temperature for each of the observed transitions. The observing parameters for the continuum observations are summarized in Table 2 .

\subsection{NANTEN Telescope}

The NANTEN telescope is a $4 \mathrm{~m}$ diameter millimeter telescope designed for a large-scale survey of molecular clouds. With this telescope we observed the emission in the $\operatorname{CO}(J=$ $1 \rightarrow 0)(v=115271.202 \mathrm{MHz})$ and $\mathrm{C}^{18} \mathrm{O}(J=1 \rightarrow 0)$ $(v=109782.173 \mathrm{MHz})$ lines during several epochs between 1999 and 2003. The half-power beam width of the telescope at $115 \mathrm{GHz}$ is 2.6 , providing a spatial resolution of $5.7 \mathrm{pc}$ at the assumed distance of the source of $7.5 \mathrm{kpc}$. The observed maps consisted in grids of 585 positions for the $\mathrm{CO}$ emission and 440 positions for the $\mathrm{C}^{18} \mathrm{O}$ emission, with spacing between observed positions of 2.5 (nearly the beam size of the instrument at the observed frequencies). The front-end was a superconductor-isolator-superconductor receiver cooled down to $4 \mathrm{~K}$ with a closed-cycle helium gas refrigerator, and the backend was an acousto-optical spectrometer with 2048 channels. The total bandwidth was $250 \mathrm{MHz}$ and the frequency resolution was $250 \mathrm{kHz}$, corresponding to a velocity resolution of $\sim 0.15 \mathrm{~km} \mathrm{~s}^{-1}$. The typical system noise 
temperature was $\sim 250 \mathrm{~K}(\mathrm{SSB})$ at $115 \mathrm{GHz}$ and $\sim 160 \mathrm{~K}$ (SSB) at $110 \mathrm{GHz}$. The main beam efficiency of the telescope is 0.89. A detailed description of the telescope and its instruments are given by Ogawa et al. (1990), Fukui et al. (1991), and Fukui \& Sakakibara (1992). Data reduction were made using the NANTEN Data Reduction Software.

\subsection{Swedish-ESO Submillimetre Telescope (SEST)}

\subsubsection{Millimeter Continuum}

The $1.2 \mathrm{~mm}$ continuum observations were carried out using the 37 channel hexagonal Sest IMaging Bolometer Array (SIMBA) during 2001. The passband of the bolometer has an equivalent width of $90 \mathrm{GHz}$ and is centered at $250 \mathrm{GHz}$ $(1200 \mu \mathrm{m})$. At the frequency band of SIMBA, the telescope has an effective beam width of $24^{\prime \prime}$ (FWHM), that provides a spatial resolution of $0.87 \mathrm{pc}$ at a distance of $7.5 \mathrm{kpc}$. The rms noise achieved is $\sim 50 \mathrm{mJy}^{\text {beam }}{ }^{-1}$. Data reduction was made using the package Mapping On-Off Pointing Skydip Infrared. The maps were calibrated from observations of Mars and Uranus and the calibration uncertainties are estimated to be lower than $20 \%$.

\subsubsection{Molecular Lines}

With SEST we observed the emission in the $\operatorname{CS}(J=5 \rightarrow$ 4) $(v=244935.644 \mathrm{MHz})$ and $\operatorname{CS}(J=2 \rightarrow 1)(v=$ 97980.968 MHz) lines during 2001 May. The half-power beam width and the main beam efficiency of the telescope were $22^{\prime \prime}$ and 0.48 at $245 \mathrm{GHz}$, and $52^{\prime \prime}$ and 0.73 at $98 \mathrm{GHz}$, respectively. The total bandwidth was $43 \mathrm{MHz}$ and the frequency resolution was $43 \mathrm{kHz}$, corresponding to a velocity resolution of $\sim 0.05 \mathrm{~km} \mathrm{~s}^{-1}$ for the $\operatorname{CS}(5 \rightarrow 4)$ observations, and a velocity resolution of $\sim 0.13 \mathrm{~km} \mathrm{~s}^{-1}$ for the $\operatorname{CS}(2 \rightarrow 1)$ observations. The typical system noise temperature was $350 \mathrm{~K}$ for $\mathrm{CS}(5 \rightarrow 4)$ observations and $250 \mathrm{~K}$ for $\mathrm{CS}(2 \rightarrow 1)$ observations. The CLASS (Continuum and Line Analysis Single-dish Software) software, part of the GILDAS package, was used for data reduction.

Figures 17-18 present maps of the observed spectra toward the G331.5-0.1 central region in the $\operatorname{CS}(2 \rightarrow 1)$ and $\operatorname{CS}(5 \rightarrow 4)$ line emission over 81 positions with a spacing of $45^{\prime \prime}$. The $\mathrm{CS}(2 \rightarrow 1)$ map then is well sampled, while the $\operatorname{CS}(5 \rightarrow 4)$ map is undersampled at this spacing, considering the smaller beam size at that frequency.

\subsection{Atacama Submillimeter Telescope Experiment}

With ASTE we observed the emission in the $\operatorname{CS}(J=7 \rightarrow 6)$ $(v=342882.950 \mathrm{MHz})$ and ${ }^{13} \mathrm{CO}(J=3 \rightarrow 2)(v=$ $330587.957 \mathrm{MHz}$ ) lines during 2005 July and 2006 July. ASTE has a beam size at $350 \mathrm{GHz}$ of $22^{\prime \prime}$ (FWHM). For both transitions 167 positions were sampled, within a $\sim 360^{\prime \prime} \times 360^{\prime \prime}$ region with $22^{\prime \prime} .5$ spacing in position-switched mode, with the OFF position located at $\alpha_{2000}=16^{\mathrm{h}} 14^{\mathrm{m}} 08^{\mathrm{s}} .4$ and $\delta_{2000}=-51^{\circ} 37^{\prime} 23^{\prime \prime}$. The system temperatures ranged between 179 and $233 \mathrm{~K}$, resulting in an rms noise antenna temperature of typically $0.1 \mathrm{~K}$ for an integration time of 4 minutes.

Main-beam temperatures, $T_{\mathrm{MB}}=T_{A}^{*} / \eta_{\mathrm{MB}}$, obtained by dividing the antenna temperature $T_{A}^{*}$ by the main-beam efficiency, $\eta_{\mathrm{MB}}$, are used throughout the analysis. During the first part of the observations, we mapped a grid of 88 positions, with $\eta_{\mathrm{MB}}=0.65$. On 2006 July, we completed the map observing 79 new positions, with $\eta_{\mathrm{MB}}=0.77$. The 1024 channels of the $350 \mathrm{GHz}$ band receiver provided a bandwidth of $128 \mathrm{MHz}$, and a channel width of $0.125 \mathrm{MHz}$. The data reduction was carried out by use of the AIPS-based software package NEWSTAR developed at NRO.

Figures 19-20 present maps of the observed spectra toward the G331.5-0.1 central region in the $\mathrm{CS}(7 \rightarrow 6)$ and ${ }^{13} \mathrm{CO}(3 \rightarrow$ 2) line emission over 167 positions with a spacing of $22^{\prime \prime} .5$ (similar to the beam size of the instrument at this frequency).

\subsection{Atacama Pathfinder Experiment}

\subsubsection{Millimeter Continuum: the ATLASGAL Survey}

The millimeter observations of the region were made as part of the APEX Telescope Large Area Survey of the Galaxy (ATLASGAL) project, which is a collaboration between the Max Planck Gesellschaft (MPG: Max Planck Institute für Radioastronomie, MPIfR Bonn, and Max Planck Institute für Astronomie, MPIA Heidelberg), the European Southern Observatory (ESO), and the Universidad de Chile. ATLASGAL is an unbiased survey of the inner region of the Galactic disk at $0.87 \mathrm{~mm}$, made using the 295 channel hexagonal array Large Apex BOlometer CAmera (LABOCA), that operates in the $345 \mathrm{GHz}$ atmospheric window, with a bandwidth of about $60 \mathrm{GHz}$. The angular resolution is 18". 6 (HPBW; corresponding to a resolution of $0.68 \mathrm{pc}$ at a distance of $7.5 \mathrm{kpc}$ ) and the total field of view is 11!.4. The survey covers an area of \pm 1.5 in galactic latitude, and $\pm 60^{\circ}$ in longitude, with a sensitivity of $50 \mathrm{mJy}^{\text {beam }}{ }^{-1}$ (Schuller et al. 2009).

\subsubsection{Molecular Lines}

Using the APEX-2a heterodyne receiver, we observed the emission in the $\operatorname{SiO}(J=7 \rightarrow 6)(v=303926.809 \mathrm{MHz})$, $\mathrm{SiO}(J=8 \rightarrow 7)(v=347330.635 \mathrm{MHz}), \operatorname{SO}\left(8_{7} \rightarrow 7_{6}\right)(v=$ $304077.844 \mathrm{MHz})$, and $\mathrm{SO}\left(8_{8} \rightarrow 7_{7}\right)(v=344310.612 \mathrm{MHz})$ lines during 2007 May. The telescope has a beam size of $17^{\prime \prime} .5$ at $345 \mathrm{GHz}$, corresponding to a spatial resolution of $0.63 \mathrm{pc}$ at a distance of $7.5 \mathrm{kpc}$. The main beam efficiency of the telescope is $\eta_{\mathrm{MB}}=0.73$ and the system temperature was $\sim 170 \mathrm{~K}$. The observations were made toward the peak position of the $\operatorname{CO}(7 \rightarrow 6)$ outflow emission $\left(\alpha_{2000}=16^{\mathrm{h}} 12^{\mathrm{m}} 10^{\mathrm{s}} .09\right.$ and $\delta_{2000}=-51^{\circ} 28^{\prime} 38^{\prime \prime} .4$ ) reported by Bronfman et al. (2008). The data were reduced using the CLASS software of the GILDAS package.

\subsection{Australia Telescope Compact Array}

The ATCA radio continuum observations were made in two epochs: 2002 November using the 6A configuration and 2005 November using the $1.5 \mathrm{C}$ configuration. These configurations utilize all six antennas and cover east-west baselines from 0.5 to $6 \mathrm{~km}$. Observations were made at the frequencies of 4.80 and $8.64 \mathrm{GHz}(6$ and $3.6 \mathrm{~cm}$ ), with a bandwidth of $128 \mathrm{MHz}$. The FWHM primary beam of ATCA at $4.8 \mathrm{GHz}$ and $8.6 \mathrm{GHz}$ are, respectively, $10^{\prime}$ and $6^{\prime}$. The total integration time in each frequency was $2.5 \mathrm{hr}$.

The calibrator PKS 1600-48 was observed before and after every on-source scan in order to correct the amplitude and phase of the interferometer data due to atmospheric and instrumental effects. The flux density was calibrated by observing PKS 1934638 (3C 84 ) for which values of $5.83 \mathrm{Jy}$ at $4.8 \mathrm{GHz}$ and $2.84 \mathrm{Jy}$ at $8.6 \mathrm{GHz}$ were adopted. Standard calibration and data reduction were performed using MIRIAD (Sault et al. 1995). Maps were made by Fourier transformation of the uniformly weighted interferometer data using the AIPS task IMAGR. The noise level in the 4.8 and $8.6 \mathrm{GHz}$ images are, respectively, 0.41 and 


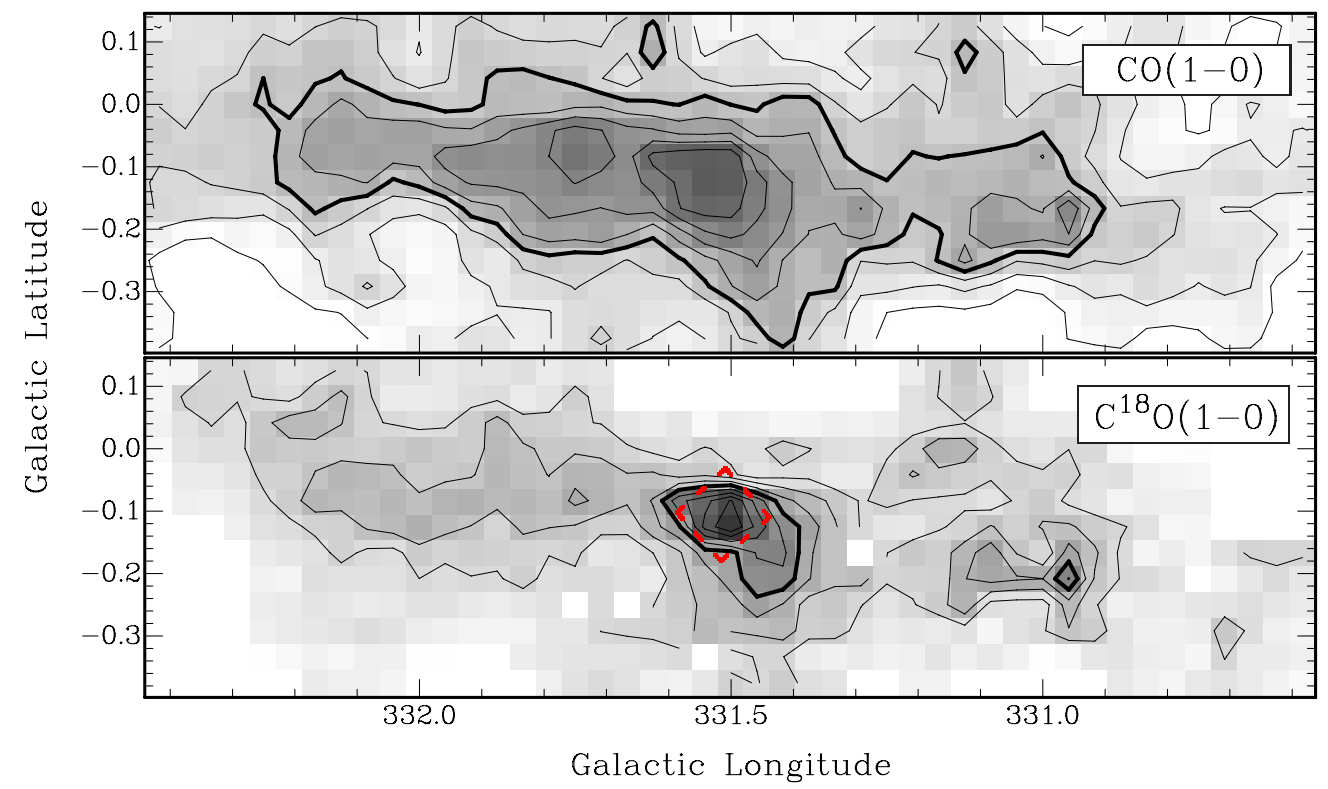

Figure 1. Maps of velocity integrated emission, in the range -117.7 to $-73.2 . \mathrm{km} \mathrm{s}^{-1}$, of the G331.5-0.1 GMC. Top: $\mathrm{CO}(1 \rightarrow 0)$. Contour levels are drawn from $20 \%(3 \sigma)$ to $90 \%$, in steps of $10 \%$, of the peak intensity $\left(297.6 \mathrm{~K} \mathrm{~km} \mathrm{~s}^{-1}\right)$. Bottom: $\mathrm{C}^{18} \mathrm{O}(1 \rightarrow 0)$. Contour levels are drawn from $20 \%(4 \sigma)$ to $90 \%$, in steps of $10 \%$, of the peak intensity $\left(19.6 \mathrm{~K} \mathrm{~km} \mathrm{~s}^{-1}\right)$. The $50 \%$ emission in each map is drawn with a thicker contour. A $7^{\prime} \times 7^{\prime}$ red dashed box, oriented in equatorial coordinates, is drawn toward the $70 \%$ emission contour in the $\mathrm{C}^{18} \mathrm{O}(1 \rightarrow 0)$ map. This area is considered as the central region of this cloud.

(A color version of this figure is available in the online journal.)

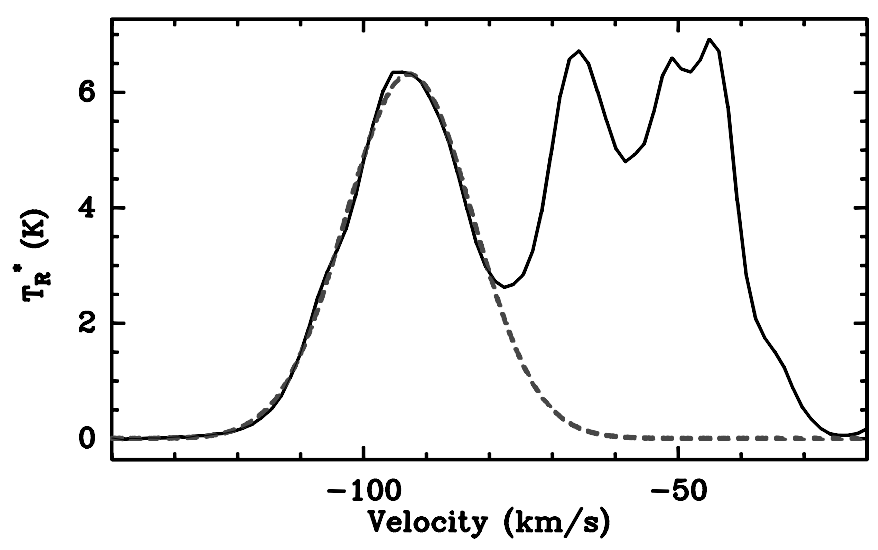

Figure 2. Average profile of the $\mathrm{CO}(1 \rightarrow 0)$ emission over the whole GMC. The dashed line corresponds to the Gaussian fit of the spectrum on the velocity range of the G331.5-0.1 GMC. The parameters of the Gaussian fit are $v_{0}=-92.8 \pm 0.006 \mathrm{~km} \mathrm{~s}^{-1}, \Delta v=23.8 \pm 0.017 \mathrm{~km} \mathrm{~s}^{-1}$, and $T_{\text {peak }}=6.31 \mathrm{~K}$.

$0.37 \mathrm{mJy}$ beam $^{-1}$. The resulting synthesized (FWHM) beams are $2 . .7 \times 11^{\prime \prime} .8$ at $4.8 \mathrm{GHz}$ and $1^{\prime \prime} .5 \times 1^{\prime \prime} .0$ at $8.6 \mathrm{GHz}$.

\section{RESULTS}

\subsection{The G331.5-0.1 Giant Molecular Cloud}

Figure 1 shows gray scale maps and contours of the velocity integrated $\mathrm{CO}$ and $\mathrm{C}^{18} \mathrm{O}$ emission between $330^{\circ} .9<l<332^{\circ} .3$ and $-0.42<b<0.14$. The range of the velocity integration is from -117.7 to $-73.2 \mathrm{~km} \mathrm{~s}^{-1}$, chosen from the average spectrum of the region (shown in Figure 2). The contours represent $20 \%$ to $90 \%$, in steps of $10 \%$, of the peak intensity of each map (297.6 K km s${ }^{-1}$ for $\mathrm{CO}, 19.6 \mathrm{~K} \mathrm{~km} \mathrm{~s}^{-1}$ for $\mathrm{C}^{18} \mathrm{O}$ ). The rms noise of the $\mathrm{CO}$ and $\mathrm{C}^{18} \mathrm{O}$ maps are $19.8 \mathrm{~K} \mathrm{~km} \mathrm{~s}^{-1}$ and $0.98 \mathrm{~K} \mathrm{~km} \mathrm{~s}^{-1}$, respectively. The emission at $50 \%$ of the peak is shown with a thicker contour in each map. The CO emission arises from an extended cloud elongated along the galactic longitude, with major and minor axes of 81'.7 and 18.9 (full width at half-power), which imply linear sizes of 178 by $41 \mathrm{pc}$ at the assumed distance of $7.5 \mathrm{kpc}$. The peak position on the CO map is located at $l=331.5, b=-0.125$, with an intensity of $297.6 \mathrm{~K} \mathrm{~km} \mathrm{~s}^{-1}$.

To estimate the kinematic distance of the G331.5-0.1 cloud, we locate it in the subcentral point at the source longitude, for the following reasons. Using the Galactic rotation curve derived by Alvarez et al. (1990), with Ro $=8.5 \mathrm{kpc}$ and $\mathrm{Vo}=220 \mathrm{~km} \mathrm{~s}^{-1}$, and a mean average velocity of $-92.8 \mathrm{~km} \mathrm{~s}^{-1}$ for the integrated GMC spectrum, the near kinematic distance would be $5.7 \mathrm{kpc}$ and the far kinematic distance $9.2 \mathrm{kpc}$. While the near distance has been preferred by Goss et al. (1972), the far distance has been considered by Kerr \& Knapp (1970). However, the GMC has two velocity components, at $-100 \mathrm{~km} \mathrm{~s}^{-1}$ and $-90 \mathrm{~km} \mathrm{~s}^{-1}$ (see Section 3.2.2), the lowest one almost reaching the terminal velocity due to pure rotation of $-110 \mathrm{~km} \mathrm{~s}^{-1}$. Caswell \& Haynes (1987) reported hydrogen recombination line absorption at $l=331.515, b=-0.069$, with a $V_{\mathrm{LSR}}$ of $-89 \mathrm{~km} \mathrm{~s}^{-1}$, as well as $\mathrm{H}_{2} \mathrm{CO}$ absorption lines at -99.8 and $-89.3 \mathrm{~km} \mathrm{~s}^{-1}$, favoring the far distance, even when they adopted the near distance following Goss et al. (1972). Nevertheless, Bronfman et al. (1996) reported emission in $\operatorname{CS}(2 \rightarrow 1)$ from the IRAS point source 16086-5119, at $l=331.552, b=-0.115$, with a $V_{\mathrm{LSR}}$ of $-100.7 \mathrm{~km} \mathrm{~s}^{-1}$, corresponding to the higher velocity component, so the continuum source for the $\mathrm{H}_{2} \mathrm{CO}$ absorption lines at both velocities is most probably the IRAS point source at $-100 \mathrm{~km} \mathrm{~s}^{-1}$, setting them both at the near distance.

Given the large spread in velocity of the integrated spectrum, the presence of two velocity components for the GMC, the lowest one almost reaching the terminal velocity, and the conflicting evidence for locating the GMC at the far or near distance, we will consider in the estimation of its physical parameters that the G331.5-0.1 cloud is located in the tangent of the Norma spiral arm, at a distance of $\sim 7.5 \mathrm{kpc}$, corresponding to the subcentral point. With this assumption, the distance of the 
GMC will have an estimated uncertainty of $\sim 30 \%$, given by the near and far kinematic positions, leading to an uncertainty in the estimation of masses and luminosities of a factor of $\sim 2$. A recent study of kinematic distances using $\mathrm{H}$ I absorption done by Jones \& Dickey (2012) puts the region G331.552-0.078, located toward the peak position of our CO emission maps, at the Norma spiral arm tangent point distance, at $7.47 \mathrm{kpc}$, a similar value to the one used in the present work.

The emission in the $\mathrm{C}^{18} \mathrm{O}(1 \rightarrow 0)$ line has a position angle of approximately $\sim 30^{\circ}$ with respect to the galactic longitude, with observed major and minor axes of 18,2 and 11'.6, respectively $(39.7 \times 25.3 \mathrm{pc}$ at a distance of $7.5 \mathrm{kpc})$. The size of the $\mathrm{C}^{18} \mathrm{O}$ structure is four times smaller than the $\mathrm{CO}$ cloud. It is necessary to keep in mind that the appearance and dimension of a molecular cloud depend strongly on the tracer used to observe it (Myers 1995). The CO observations most likely trace all the gas within the GMC, including low and high column density gas, while $\mathrm{C}^{18} \mathrm{O}$ is tracing only the high column density gas. The $\mathrm{C}^{18} \mathrm{O}$ peak position coincides with the peak emission in the $\mathrm{CO}(1 \rightarrow 0)$ line. Fitting Gaussian profiles to the spectra observed at $l=331.5, b=-0.125$, we determine $V_{\mathrm{lsr}}=-89.7 \mathrm{~km} \mathrm{~s}^{-1}$ and $\Delta V(\mathrm{FWHM})=3.8 \mathrm{~km} \mathrm{~s}^{-1}$ for CO, and $V_{\mathrm{lsr}}=-89 \mathrm{~km} \mathrm{~s}^{-1}$ and $\Delta V(\mathrm{FWHM})=2.9 \mathrm{~km} \mathrm{~s}^{-1}$ for the $\mathrm{C}^{18} \mathrm{O}$ emission.

Using the $\mathrm{C}^{18} \mathrm{O}(1 \rightarrow 0)$ integrated map, we define the central region of the G331.5-0.1 GMC as the region that emits more than seventy percent of the peak integrated emission (red dashed box in Figure 1). This area is $7^{\prime}$ in size $(\sim 15 \mathrm{pc}$ at the source distance) and it is centered at $l=331.523, b=-0.099$ $\left(\alpha_{2000}=16^{\mathrm{h}} 12^{\mathrm{m}} 12^{\mathrm{s}}\right.$ and $\left.\delta_{2000}=-51^{\circ} 28^{\prime} 00^{\prime \prime}\right)$. The central region is a single structure coherent in velocity, as it can be seen on the position-velocity maps for $\mathrm{CO}$ and $\mathrm{C}^{18} \mathrm{O}$ (Figure 3). Further observations with better angular resolution have shown that this region has two velocity components, with a difference of $\sim 12 \mathrm{~km} \mathrm{~s}^{-1}$ (see Section 3.2.2).

Figure 4 shows a map of the emission observed at $870 \mu \mathrm{m}$ by ATLASGAL between $331^{\circ}<l<332^{\circ}$ and $-0.4<b<0.15$. Overlaid is a velocity integrated $\mathrm{C}^{18} \mathrm{O}$ emission map. The range of velocity integration and the emission contours are the same as for Figure 1. The $0.87 \mathrm{~mm}$ map also shows the position of 13 sources ( 2 of them are double sources) from the RMS catalog in the proximity of the tangent area of the Norma spiral arm. Clearly there is a concentration of dust continuum emission sources toward the defined central region of the cloud (red dashed box in Figure 4). Six millimeter-wave compact sources were identified inside the defined G331.5-0.1 GMC central region, and three of them are associated to RMS sources. This suggests an active formation of massive stars within the central region of G331.5-0.1 GMC.

\subsection{The G331.5-0.1 GMC Central Region}

In this section, we present a detailed study of millimeter continuum and molecular line observation toward the $7^{\prime} \times 7^{\prime}$ area defined in the previous section as the G331.5-0.1 GMC central region. The beam sizes of the observations $\left(\sim 30^{\prime \prime}\right)$ are approximately five times better than the observations of $\mathrm{CO}(1 \rightarrow 0)$ and $\mathrm{C}^{18} \mathrm{O}(1 \rightarrow 0)$, and they give information in the $\sim 1$ pc at a distance of $7.5 \mathrm{kpc}$. The maps of G331.5-0.1 GMC have been presented so far in galactic coordinates, mainly to show the extension of this region in galactic longitude. Nevertheless, we present our observations here and following sections in a more traditional style using equatorial coordinates.

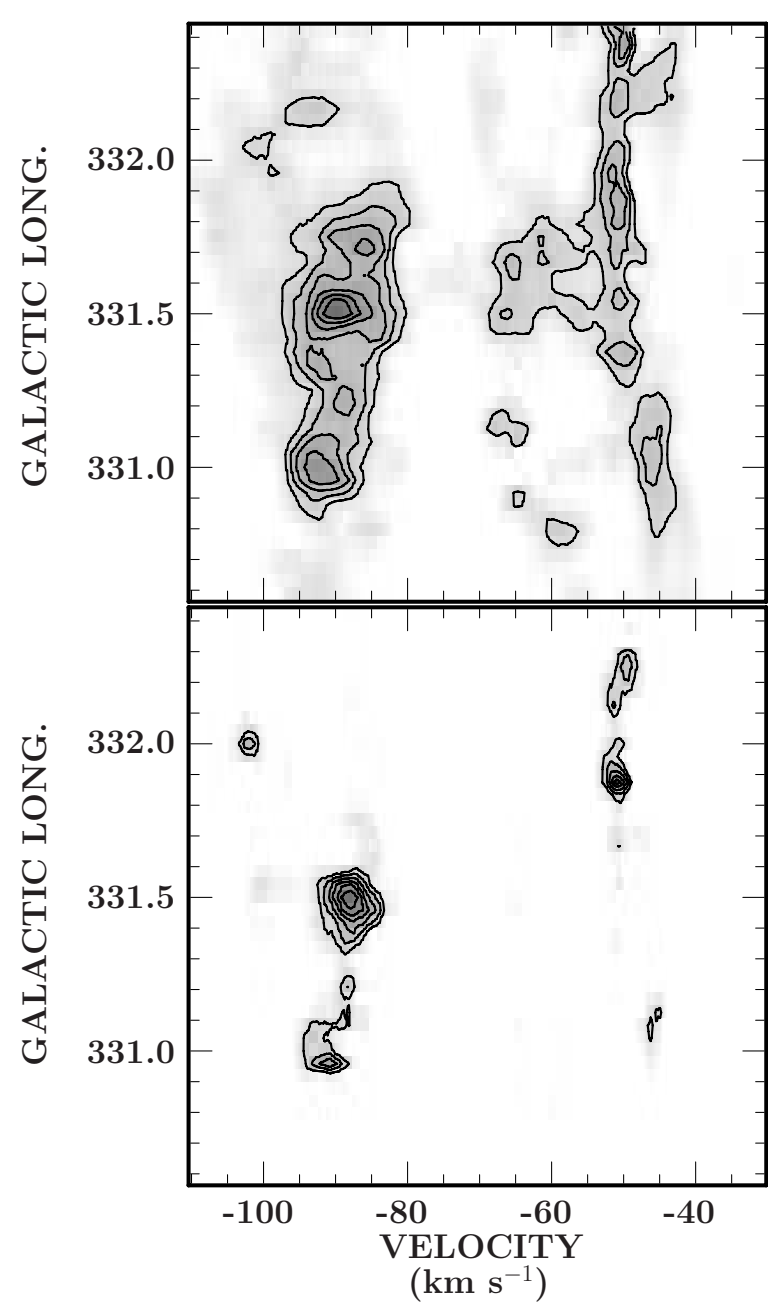

Figure 3. Position-velocity maps of the $\mathrm{CO}(1 \rightarrow 0)$ (top) and $\mathrm{C}^{18} \mathrm{O}(1 \rightarrow 0)$ emission (bottom) integrated between -0.21 and -0.04 in Galactic Latitude. Contour levels are drawn at $40 \%$ to $90 \%$, in steps of $10 \%$, of the peak intensity $\left(11.5 \mathrm{~K} \mathrm{~km} \mathrm{~s}^{-1}\right.$ for $\mathrm{CO}$ and $0.78 \mathrm{~K} \mathrm{~km} \mathrm{~s}^{-1}$ for $\left.\mathrm{C}^{18} \mathrm{O}\right)$. The $\mathrm{C}^{18} \mathrm{O}$ emission shows a clear component between $l=331.3$ and $l=331.6$ at $\sim-90 \mathrm{~km} \mathrm{~s}^{-1}$.

\subsubsection{Dust Emission: Millimeter Continuum Observations}

Figure 5 presents maps of the $1.2 \mathrm{~mm}$ and $0.87 \mathrm{~mm}$ dust continuum emission observed with the SEST and APEX telescopes respectively. They reveal the presence of six dust continuum substructures with strong emission. We labeled these sources as MM1, MM2, MM3, MM4, MM5, and MM6, according to increasing order in right ascension. The observed parameters of the millimeter sources are given in Table 3. Columns 3 and 4 give their peak position. Columns 5 and 6 give the peak flux density and the total flux density, respectively, the latter measured with the AIPS task IMEAN. Column 7 give the deconvolved major and minor FWHM angular sizes obtained from Gaussian fits to the observed spatial distribution. Column 8 gives the physical size of the sources, obtained from the geometric mean of the angular size obtained at $1.2 \mathrm{~mm}$ and $0.87 \mathrm{~mm}$, and considering a distance to the sources of $7.5 \mathrm{kpc}$. The average size of the millimeter sources within the G331.5-0.1 central region is $1.6 \mathrm{pc}$. Following the description of Williams et al. (2000), we refer to these millimeter-wave structures as clumps.

\subsubsection{Molecular Line Emission}

The observed parameters of the line emission detected at the peak position of the millimeter clumps are given in Table 4 . 


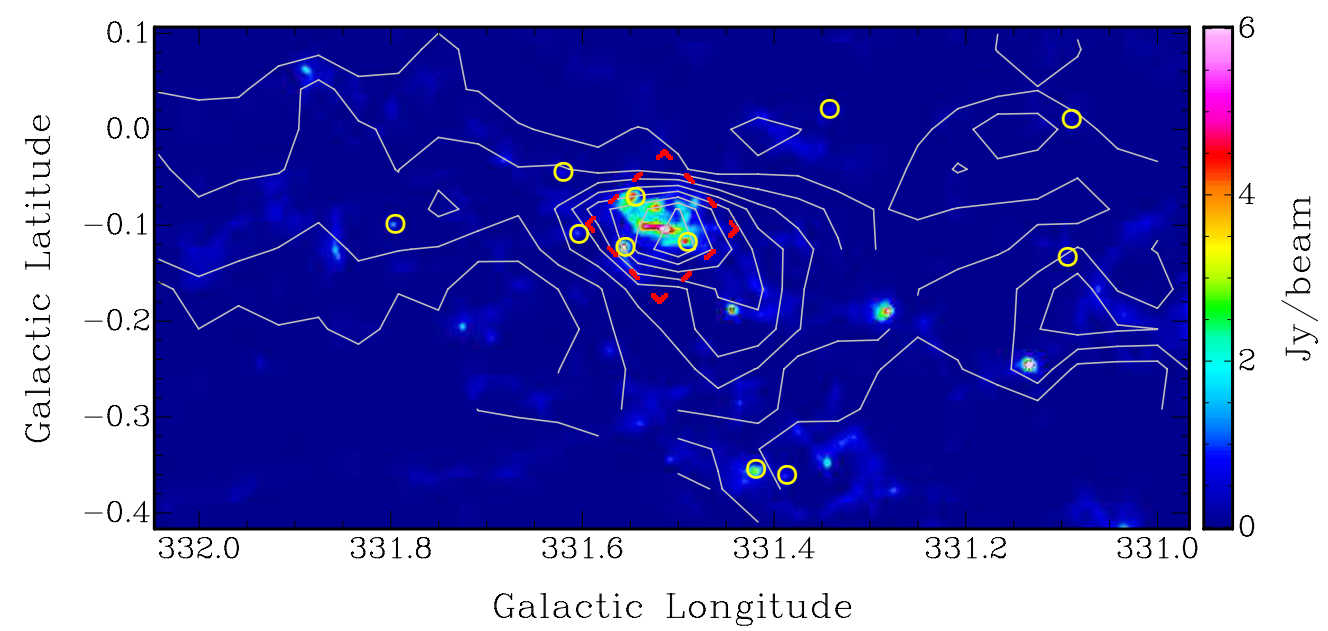

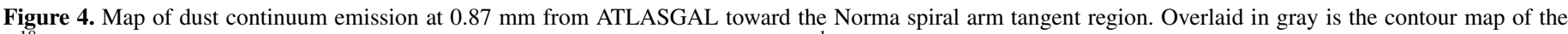

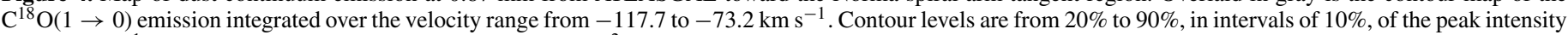

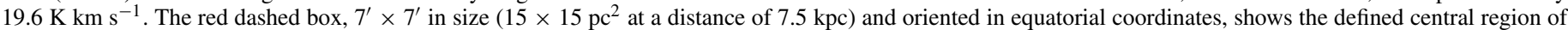

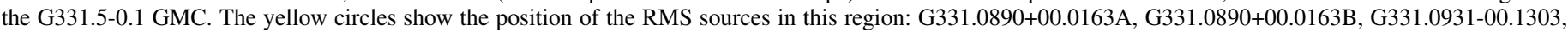

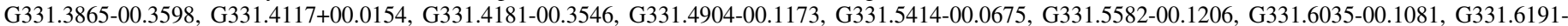
00.0442A, G331.6191-00.0442B, and G331.7953-00.0979.

Table 3

Observed Parameters of Dust Continuum Emission

\begin{tabular}{|c|c|c|c|c|c|c|c|}
\hline Clump & $\begin{array}{c}\lambda \\
(\mu \mathrm{m})\end{array}$ & $\alpha(\mathrm{J} 2000)$ & $\delta(\mathrm{J} 2000)$ & $\begin{array}{l}\text { Peak Flux Density } \\
\left(\mathrm{Jy} \mathrm{beam}^{-1}\right)\end{array}$ & $\begin{array}{c}\text { Flux Density } \\
(\mathrm{Jy})\end{array}$ & $\begin{array}{c}\text { Angular Size } \\
\left({ }^{\prime \prime}\right)\end{array}$ & $\begin{array}{l}\text { Diameter } \\
(\mathrm{pc})\end{array}$ \\
\hline \multirow[t]{2}{*}{ MM1 } & 1200 & 161207.69 & -513007.1 & 1.2 & 3.1 & $36 \times 32$ & 1.3 \\
\hline & 870 & 161207.57 & -513003.5 & 5.0 & 27.4 & $44 \times 31$ & \\
\hline \multirow[t]{2}{*}{ MM2 } & 1200 & 161208.43 & -512711.1 & 1.2 & 6.4 & $62 \times 40$ & 1.9 \\
\hline & 870 & 161206.90 & -512715.6 & 4.5 & 38.3 & $59 \times 49$ & \\
\hline \multirow[t]{2}{*}{ MM3 } & 1200 & 161209.34 & -512839.0 & 2.9 & 11.6 & $50 \times 34$ & 1.2 \\
\hline & 870 & 161210.13 & -512839.5 & 13.8 & 66.7 & $36 \times 30$ & \\
\hline \multirow[t]{2}{*}{ MM4 } & 1200 & 161210.08 & -512551.0 & 1.2 & 4.9 & $56 \times 38$ & 1.9 \\
\hline & 870 & 161210.73 & -512545.5 & 4.6 & 42.7 & $72 \times 45$ & \\
\hline \multirow[t]{2}{*}{ MM5 } & 1200 & 161214.45 & -512742.6 & 1.2 & 7.6 & $76 \times 34$ & 1.9 \\
\hline & 870 & 161215.25 & -512739.41 & 5.5 & 52.5 & $69 \times 38$ & \\
\hline \multirow[t]{2}{*}{ MM6 } & 1200 & 161227.28 & -512741.8 & 1.6 & 3.5 & $32 \times 32$ & 1.2 \\
\hline & 870 & 161227.45 & -512739.1 & 8.0 & 27.6 & $30 \times 21$ & \\
\hline
\end{tabular}

Also given are the parameters of an average spectrum of each clump for the $\mathrm{CS}(7 \rightarrow 6)$ and ${ }^{13} \mathrm{CO}(3 \rightarrow 2)$ lines, obtained from a three by three map centered at the peak position of each clump. For non-Gaussian profiles, the velocity spread $\Delta V$ is estimated from $\Delta V=I\left(T_{A}^{*}\right) / T_{A}^{*}$ Peak. The equations for $I\left(T_{A}^{*}\right)$ and its associated error $\sigma_{I\left(T_{A}^{*}\right)}^{2}$ are obtained from (e.g., Shirley et al. 2003)

$$
\begin{aligned}
& I\left(T_{A}^{*}\right)=\int_{v_{1}}^{v_{2}} T_{A}^{*} d v \\
& \sigma_{I\left(T_{A}^{*}\right)}^{2}=\left\langle\sqrt{\Delta v_{\text {line }} \delta v_{\text {chan }}} \sigma_{T_{A}^{*}}\right\rangle_{\text {map }}^{2}+\sigma_{\text {base }}^{2},
\end{aligned}
$$

with $\Delta v_{\text {line }}=v_{2}-v_{1}$ is the full velocity range of the line emission, $\delta v_{\text {chan }}$ is the velocity resolution of the spectrometer, and $\sigma_{T_{A}^{*}}$ is the uncertainty in the antenna temperature. The additional error contribution $\sigma_{\text {base }}$, from residual variation in the baseline due to linear baseline subtraction, was not considered. The error $\sigma_{I\left(T_{A}^{*}\right)}$ was propagated into the uncertainty in $\Delta V$.

Figure 6 shows the spectra toward the peak intensity position of the clumps MM1 through MM6 in the molecular lines $\mathrm{CS}(2 \rightarrow 1), \mathrm{CS}(5 \rightarrow 4), \mathrm{CS}(7 \rightarrow 6)$ and ${ }^{13} \mathrm{CO}(3 \rightarrow 2)$.

We discovered in the $\mathrm{CS}(7 \rightarrow 6)$ map the presence of a broad and strong wing emission toward the peak position of the brightest $1.2 \mathrm{~mm}$ clump (MM3), at $\alpha_{2000}=16^{\mathrm{h}} 12^{\mathrm{m}} 10^{\mathrm{s}} 13$ and $\delta_{2000}=-51^{\circ} 28^{\prime} 37^{\prime \prime} .5$. The characteristics of this spectrum, along with other $\mathrm{CO}$ emission lines, were previously reported by Bronfman et al. (2008). Their analysis indicates that it corresponds to an unresolved, energetic, and massive molecular outflow (flow mass of $\sim 55 M_{\odot}$; momentum of $\sim 2.4 \times$ $10^{3} M_{\odot} \mathrm{km} \mathrm{s}^{-1}$; kinetic energy of $\sim 1.4 \times 10^{48}$ ergs).

The molecular transitions $\mathrm{SiO}(7 \rightarrow 6), \mathrm{SiO}(8 \rightarrow 7)$, $\mathrm{SO}\left(8_{7} \rightarrow 7_{6}\right)$, and $\mathrm{SO}\left(8_{8} \rightarrow 7_{7}\right)$ were observed toward the position of the molecular outflow. SO and $\mathrm{SiO}$ trace shocked gas toward dense cores (see Gottlieb et al. 1978; Rydbeck et al. 1980; Swade 1989; Miettinen et al. 2006) where the density is high enough to excite it. High-velocity $\mathrm{SiO}$ and $\mathrm{SO}$ has been observed in some of the most powerful molecular outflows (e.g., Welch et al. 1981; Plambeck et al. 1982; Martin-Pintado et al. 1992), and our current understanding is that $\mathrm{SiO}$ and $\mathrm{SO}$ are evaporated from the dust grains when the shock velocity is greater than about $20 \mathrm{~km} \mathrm{~s}^{-1}$, depending on the composition of the grain-mantle in the preshock gas (e.g., Schilke et al. 1997). Figure 7 shows the spectra of the $\mathrm{SO}$ and $\mathrm{SiO}$ lines.

Line profiles of the CS and CO emission show the presence of two distinct velocity components centered at $\sim-88.9$ and $\sim-100.8 \mathrm{~km} \mathrm{~s}^{-1}$ (see Figure 6). Maps of the velocity integrated emission in the ranges of -93.9 to $-84.4 \mathrm{~km} \mathrm{~s}^{-1}$ 

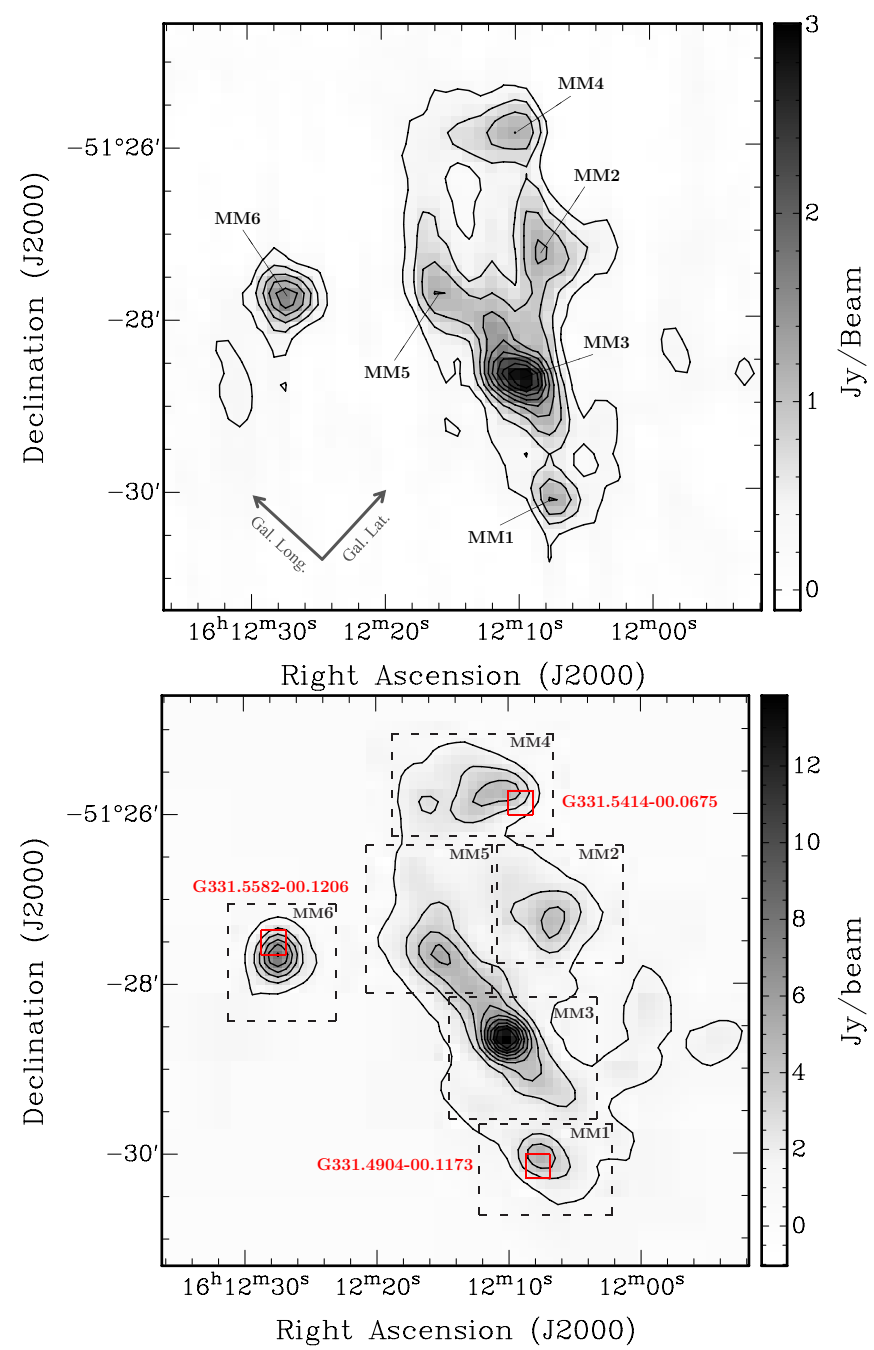

Figure 5. Maps of the dust continuum emission of the central region of the G331.5-0.1 GMC. Top: map of the $1.2 \mathrm{~mm}$ emission observed with SIMBA. The angular resolution is $24^{\prime \prime}$. Contour levels are from $10 \%(3 \sigma)$ to $90 \%$, in steps of $10 \%$, of the peak intensity of $2.9 \mathrm{Jy}_{\text {beam }^{-1}}$. The peak position of millimeter clumps MM1-MM6, and the Galactic latitude and longitude directions are shown in this map. Bottom: map of the $0.87 \mathrm{~mm}$ emission observed with LABOCA. The angular resolution is $18^{\prime \prime} .6$. Contours levels are from $10 \%(4 \sigma)$ to $90 \%$, in steps of $10 \%$, of the peak intensity of $13.8 \mathrm{Jy}^{\text {beam }}{ }^{-1}$. Dashed boxes in this map show the considered area of each millimeter clump for spectral energy distribution analysis. Red boxes mark the position of RMS sources in the region. The size of these red boxes is $18^{\prime \prime} \times 18^{\prime \prime}$ to account for the beam size of the RMS catalog.

(A color version of this figure is available in the online journal.)

and -105.4 to $-95 \mathrm{~km} \mathrm{~s}^{-1}$ are presented in Figures 8 and 9. Clumps MM1 and MM6 are associated with the lowest velocity component $\left(\sim-100.8 \mathrm{~km} \mathrm{~s}^{-1}\right)$, while clumps MM2, MM3, MM4, and MM5 are associated with the high velocity component $\left(\sim-88.9 \mathrm{~km} \mathrm{~s}^{-1}\right)$. There is a good correspondence between $\mathrm{CS}(7 \rightarrow 6)$ map and the dust continuum emissions (see Figure 10), indicating that these two tracers are probes of regions with similar physical conditions. We will refer to the region including the four high-velocity clumps as the "complex of clumps" in the G331.5-0.1 GMC central region.

The angular sizes of the structures defined by the integration of the $\operatorname{CS}(7 \rightarrow 6)$ line and associated to the clumps, and the nominal radii resulting from the deconvolution of the telescope beam size, are summarized in Table 5. For MM3, the $\mathrm{CS}(7 \rightarrow 6)$ peak position and average spectra are well fitted with two Gaussian components. The first component corresponds to the broad wind emission, associated with the high-velocity molecular outflow, while the second Gaussian component corresponds to the ambient gas emission. For the peak position spectrum, the Gaussian fit of the wing emission has $T_{A}^{*}=0.92 \pm 0.12 \mathrm{~K}$ and $\Delta v=31.1 \pm 2.8 \mathrm{~km} \mathrm{~s}^{-1}$ (FWHM), and the ambient gas Gaussian fitting has $T_{A}^{*}=1.40 \pm 0.12 \mathrm{~K}$ and $\Delta v=6.1 \pm 0.1 \mathrm{~km} \mathrm{~s}^{-1}$ (FWHM).

\subsubsection{Mid-infrared and Far-infrared Emission}

Infrared observations are a useful tool to study the process of massive star formation. At these wavelengths, radiation is less affected by extinction than at visible wavelengths; therefore infrared observations can give us information of the nearby environment of newly formed, massive stars that are surrounded and obscured by cool dust. The mid-infrared (range 5-25 $\mu \mathrm{m}$ ) and far-infrared (range 25-400 $\mu \mathrm{m}$ ) observations are dominated by the thermal emission from dust that reradiates the absorbed UV radiation from newly born OB stars and reprocessed photons from the ionized nebula. In the mid-infrared range the contribution from polycyclic aromatic hydrocarbons, from which emission is strong in photo-dissociation regions, is also significant.

Figure 11 shows a three-color infrared image made using data from the IRAC bands at $3.6 \mu \mathrm{m}$ (blue), $4.5 \mu \mathrm{m}$ (green) and $8.0 \mu \mathrm{m}$ (red), overlaid with contours of the emission at $0.87 \mathrm{~mm}$. While the infrared emission agrees with the millimeter emission in the global sense, the $8.0 \mu \mathrm{m}$ emission seems to trace the envelope of a ionized region, with the millimeter emission of the complex of clumps surrounding this structure. Also, we find strong and compact emission associated with the MM3 clump in the three IRAC bands, with an angular size of $\sim 14^{\prime \prime}$.

For each millimeter clump, we measured the integrated flux densities in the four Spitzer IRAC bands $(8.0 \mu \mathrm{m}, 5.8 \mu \mathrm{m}$, $4.5 \mu \mathrm{m}$, and $3.6 \mu \mathrm{m}$; Fazio et al. 2004), on the $M S X$ bands $(21.3 \mu \mathrm{m}, 14.7 \mu \mathrm{m}, 12.1 \mu \mathrm{m}$, and $8.3 \mu \mathrm{m}$; Mill et al. 1994) and IRAC bands (100 $\mu \mathrm{m}, 60 \mu \mathrm{m}, 25 \mu \mathrm{m}$, and $12 \mu \mathrm{m}$; Neugebauer et al. 1984). The results are tabulated in Table 6.

\subsection{High Angular Resolution Observations of the G331.5-0.1 GMC Central Region}

We present in this section interferometric observations of radio continuum emission toward the G331.5-0.1 GMC central region. The beam size of the observations $\left(\sim 2^{\prime \prime}\right)$ allow us to study compact structures at sub-parsec scales. The analysis also considered association between the radio continuum components found in recent $\mathrm{OH}$ and methanol maser catalogs at high resolution.

\subsubsection{Radio Continuum Emission}

Figure 12 shows contour maps of the radio continuum emission at 6 and $3.6 \mathrm{~cm}$ toward the central region of G331.50.1 GMC. Our observations reveal the presence of four distinct compact radio sources within the mapped field of $10^{\prime}$, labeled as components A, B, C, and D, and associated with the clumps MM1, MM4, MM3, and MM6, respectively (see Figure 11). The positions, flux densities and beam deconvolved sizes (FWHM) of the sources, obtained with the AIPS task IMFIT, are given in Table 7 .

Components A, B, and D are also associated, respectively, with the G331.4904-00.1173, G331.5414-00.0675, and G331.5582-00.1206 RMS sources candidates to MYSOs (Urquhart et al. 2008). The detection of radio emission indicate that these three candidates are UCHII regions and therefore 
Table 4

Molecular Lines: Observed Parameters

\begin{tabular}{|c|c|c|c|c|c|c|}
\hline \multirow[t]{2}{*}{ Line } & \multicolumn{3}{|c|}{ Peak } & \multicolumn{3}{|c|}{ Average } \\
\hline & $\begin{array}{l}T_{A}^{*} \\
(\mathrm{~K})\end{array}$ & $\begin{array}{c}V \\
\left(\mathrm{~km} \mathrm{~s}^{-1}\right)\end{array}$ & $\begin{array}{c}\Delta v \\
\left(\mathrm{~km} \mathrm{~s}^{-1}\right)\end{array}$ & $\begin{array}{l}T_{A}^{*} \\
(\mathrm{~K})\end{array}$ & $\begin{array}{c}V \\
\left(\mathrm{~km} \mathrm{~s}^{-1}\right)\end{array}$ & $\begin{array}{c}\Delta v \\
\left(\mathrm{~km} \mathrm{~s}^{-1}\right)\end{array}$ \\
\hline \multicolumn{7}{|c|}{ MM1 } \\
\hline \multirow[t]{2}{*}{$\mathrm{CS}(2 \rightarrow 1)$} & $0.896 \pm 0.052$ & $-102.39 \pm 0.03$ & $5.7 \pm 0.1$ & $\cdots$ & $\cdots$ & $\cdots$ \\
\hline & $0.734 \pm 0.052$ & $-88.06 \pm 0.05$ & $5.7 \pm 0.1$ & $\cdots$ & $\cdots$ & $\cdots$ \\
\hline \multirow[t]{2}{*}{$\mathrm{CS}(5 \rightarrow 4)$} & $0.134 \pm 0.073$ & $-102.31 \pm 0.20$ & $5.2 \pm 0.5$ & $\cdots$ & $\cdots$ & $\cdots$ \\
\hline & $0.181 \pm 0.073$ & $-88.43 \pm 0.13$ & $3.1 \pm 0.4$ & $\cdots$ & $\ldots$ & $\cdots$ \\
\hline $\mathrm{CS}(7 \rightarrow 6)$ & $0.634 \pm 0.084$ & $-102.42 \pm 0.07$ & $4.6 \pm 0.2$ & $0.148 \pm 0.035$ & $-102.21 \pm 0.11$ & $4.3 \pm 0.3$ \\
\hline \multirow[t]{2}{*}{${ }^{13} \mathrm{CO}(3 \rightarrow 2)$} & $6.466 \pm 0.110$ & $-101.30 \pm 0.03^{\mathrm{a}}$ & $5.2 \pm 0.1^{\mathrm{b}}$ & $3.283 \pm 0.046$ & $-101.32 \pm 0.03^{\mathrm{a}}$ & $5.1 \pm 0.1^{b}$ \\
\hline & $2.442 \pm 0.110$ & $-87.78 \pm 0.04$ & $6.0 \pm 0.1$ & $3.246 \pm 0.046$ & $-87.94 \pm 0.03^{\mathrm{a}}$ & $6.2 \pm 0.1^{\mathrm{b}}$ \\
\hline \multicolumn{7}{|c|}{ MM2 } \\
\hline $\mathrm{CS}(2 \rightarrow 1)$ & $1.591 \pm 0.053$ & $-89.67 \pm 0.02$ & $4.3 \pm 0.1$ & $\cdots$ & $\cdots$ & $\cdots$ \\
\hline $\mathrm{CS}(5 \rightarrow 4)$ & $0.738 \pm 0.083$ & $-89.65 \pm 0.03$ & $3.6 \pm 0.1$ & $\ldots$ & $\ldots$ & $\ldots$ \\
\hline $\mathrm{CS}(7 \rightarrow 6)$ & $1.080 \pm 0.120$ & $-89.72 \pm 0.05$ & $4.5 \pm 0.1$ & $0.680 \pm 0.049$ & $-89.76 \pm 0.04$ & $4.4 \pm 0.1$ \\
\hline${ }^{13} \mathrm{CO}(3 \rightarrow 2)$ & $10.753 \pm 0.106$ & $-88.93 \pm 0.03^{\mathrm{a}}$ & $6.1 \pm 0.1^{\mathrm{b}}$ & $9.386 \pm 0.067$ & $-89.40 \pm 0.01$ & $5.5 \pm 0.1$ \\
\hline \multicolumn{7}{|c|}{ MM3 } \\
\hline $\mathrm{CS}(2 \rightarrow 1)$ & $2.650 \pm 0.067$ & $-89.21 \pm 0.01$ & $5.3 \pm 0.1$ & $\cdots$ & $\cdots$ & $\cdots$ \\
\hline $\mathrm{CS}(5 \rightarrow 4)$ & $1.033 \pm 0.086$ & $-88.89 \pm 0.03^{\mathrm{a}}$ & $5.0 \pm 0.4^{\mathrm{b}}$ & $\ldots$ & $\ldots$ & $\ldots$ \\
\hline \multirow[t]{2}{*}{$\mathrm{CS}(7 \rightarrow 6)$} & $1.398 \pm 0.120$ & $-90.24 \pm 0.05$ & $6.1 \pm 0.1^{\mathrm{c}}$ & $0.422 \pm 0.048$ & $-89.68 \pm 0.06$ & $6.0 \pm 0.2^{\mathrm{d}}$ \\
\hline & $0.916 \pm 0.120$ & $-91.79 \pm 2.46$ & $31.1 \pm 2.8^{\mathrm{c}}$ & $0.146 \pm 0.049$ & $-91.91 \pm 0.40$ & $26.3 \pm 0.9^{\mathrm{d}}$ \\
\hline \multirow[t]{2}{*}{${ }^{13} \mathrm{CO}(3 \rightarrow 2)$} & $0.996 \pm 0.110$ & $-99.93 \pm 0.07$ & $6.3 \pm 0.2$ & $0.770 \pm 0.051$ & $-100.38 \pm 0.01$ & $4.1 \pm 0.1$ \\
\hline & $15.915 \pm 0.110$ & $-89.44 \pm 0.01$ & $6.8 \pm 0.1$ & $11.869 \pm 0.051$ & $-88.98 \pm 0.03^{\mathrm{a}}$ & $6.0 \pm 0.1^{\mathrm{b}}$ \\
\hline $\mathrm{SO}\left(8_{7} \rightarrow 7_{6}\right)$ & $2.709 \pm 0.042$ & $-90.20 \pm 0.03^{\mathrm{a}}$ & $29.8 \pm 0.5^{\mathrm{b}}$ & $\ldots$ & $\ldots$ & $\ldots$ \\
\hline $\mathrm{SO}\left(8_{8} \rightarrow 7_{7}\right)$ & $2.024 \pm 0.046$ & $-90.31 \pm 0.03^{\mathrm{a}}$ & $32.6 \pm 0.6^{\mathrm{b}}$ & $\ldots$ & $\ldots$ & $\ldots$ \\
\hline $\mathrm{SiO}(7 \rightarrow 6)$ & $0.859 \pm 0.035$ & $-89.31 \pm 0.03^{\mathrm{a}}$ & $43.3 \pm 1.8^{\mathrm{b}}$ & $\cdots$ & $\cdots$ & $\cdots$ \\
\hline$\underline{\mathrm{SiO}(8 \rightarrow 7)}$ & $0.899 \pm 0.045$ & $-89.64 \pm 0.03^{\mathrm{a}}$ & $47.2 \pm 2.4^{\mathrm{b}}$ & $\cdots$ & $\cdots$ & $\cdots$ \\
\hline \multicolumn{7}{|c|}{ MM4 } \\
\hline $\mathrm{CS}(2 \rightarrow 1)$ & $2.262 \pm 0.055$ & $-88.34 \pm 0.01$ & $4.7 \pm 0.1$ & $\ldots$ & $\ldots$ & $\ldots$ \\
\hline $\mathrm{CS}(5 \rightarrow 4)$ & $0.776 \pm 0.064$ & $-89.17 \pm 0.03$ & $4.6 \pm 0.1$ & $\ldots$ & $\ldots$ & $\ldots$ \\
\hline $\mathrm{CS}(7 \rightarrow 6)$ & $1.498 \pm 0.120$ & $-88.21 \pm 0.04$ & $3.9 \pm 0.1$ & $0.413 \pm 0.046$ & $-88.83 \pm 0.06$ & $4.6 \pm 0.1$ \\
\hline${ }^{13} \mathrm{CO}(3 \rightarrow 2)$ & $15.320 \pm 0.090$ & $-87.79 \pm 0.01$ & $4.5 \pm 0.1$ & $8.616 \pm 0.047$ & $-88.33 \pm 0.01$ & $5.1 \pm 0.1$ \\
\hline \multicolumn{7}{|c|}{ MM5 } \\
\hline $\mathrm{CS}(2 \rightarrow 1)$ & $2.949 \pm 0.056$ & $-88.44 \pm 0.01$ & $5.1 \pm 0.1$ & $\ldots$ & $\ldots$ & $\ldots$ \\
\hline $\mathrm{CS}(5 \rightarrow 4)$ & $1.057 \pm 0.085$ & $-88.89 \pm 0.03$ & $4.7 \pm 0.1$ & $\ldots$ & $\ldots$ & $\ldots$ \\
\hline $\operatorname{CS}(7 \rightarrow 6)$ & $0.899 \pm 0.130$ & $-89.21 \pm 0.07$ & $4.5 \pm 0.2$ & $0.463 \pm 0.048$ & $-88.76 \pm 0.05$ & $4.9 \pm 0.1$ \\
\hline${ }^{13} \mathrm{CO}(3 \rightarrow 2)$ & $0.701 \pm 0.100$ & $-100.32 \pm 0.05$ & $2.1 \pm 0.1$ & $0.714 \pm 0.066$ & $-100.54 \pm 0.02$ & $2.9 \pm 0.05$ \\
\hline & $19.143 \pm 0.100$ & $-88.41 \pm 0.03^{\mathrm{a}}$ & $5.4 \pm 0.1^{\mathrm{b}}$ & $14.312 \pm 0.066$ & $-88.07 \pm 0.01$ & $5.2 \pm 0.1$ \\
\hline \multicolumn{7}{|c|}{ MM6 } \\
\hline $\mathrm{CS}(2 \rightarrow 1)$ & $1.039 \pm 0.075$ & $-100.48 \pm 0.04$ & $4.5 \pm 0.1$ & $\ldots$ & $\ldots$ & $\ldots$ \\
\hline $\mathrm{CS}(5 \rightarrow 4)$ & $0.205 \pm 0.073$ & $-100.35 \pm 0.12$ & $4.0 \pm 0.3$ & $\ldots$ & $\ldots$ & $\ldots$ \\
\hline $\operatorname{CS}(7 \rightarrow 6)$ & $1.837 \pm 0.099$ & $-100.05 \pm 0.03$ & $5.7 \pm 0.1$ & $0.423 \pm 0.045$ & $-100.00 \pm 0.05$ & $6.3 \pm 0.1$ \\
\hline${ }^{13} \mathrm{CO}(3 \rightarrow 2)$ & $10.806 \pm 0.110$ & $-99.84 \pm 0.02$ & $6.2 \pm 0.1$ & $5.684 \pm 0.042$ & $-100.48 \pm 0.03^{\mathrm{a}}$ & $5.6 \pm 0.1^{b}$ \\
\hline
\end{tabular}

Notes.

${ }^{a}$ The error in the estimation of the velocity is fixed to $\sigma_{V}=0.03$.

${ }^{\mathrm{b}}$ Non-Gaussian profile. $\Delta V=I\left(T_{A}^{*}\right) / T_{A}^{*}$ Peak.

${ }^{\mathrm{c}}$ For this spectrum, we considered two Gaussian fittings. The first is related with the broad emission related with outflowing gas, and the second Gaussian fit is made to the spectrum after the subtraction of the broad emission and is related with the ambient gas.

${ }^{\mathrm{d}}$ Same as previous note, but for the composited integrated spectrum.

related with a more evolved state than a genuine MYSOs. The brightest radio source (component $\mathrm{C}$ ) is associated with the very energetic outflow detected toward the G331.5-0.1 central region (Bronfman et al. 2008). Figure 13 shows the radio spectra of all four components. Components $\mathrm{B}$ and $\mathrm{C}$ exhibit an increasing flux density with frequency, thus they could be optically thick ultracompact regions or stellar wind sources. The dotted line corresponds to the best fit of the spectral energy distribution (SED) assuming that they correspond to regions of ionized gas with uniform density, and that the emission can be modeled as a modified blackbody, with $I_{v}=B_{v}\left(T_{e}\right)\left[1-\exp \left(-\tau_{v}\right)\right]$ and the opacity $\tau$ estimated from (Wilson et al. 2009):

$$
\tau_{v}=8.235 \times 10^{-2}\left(\frac{T_{e}}{\mathrm{~K}}\right)^{-1.35}\left(\frac{v}{\mathrm{GHz}}\right)^{-2.1}\left(\frac{\mathrm{EM}}{\mathrm{pc} \mathrm{cm}^{-6}}\right),
$$

with $T_{e}$ the electron temperature and EM the emission measure. We considered $T_{e}=10^{4} \mathrm{~K}$. The derived spectral indexes 

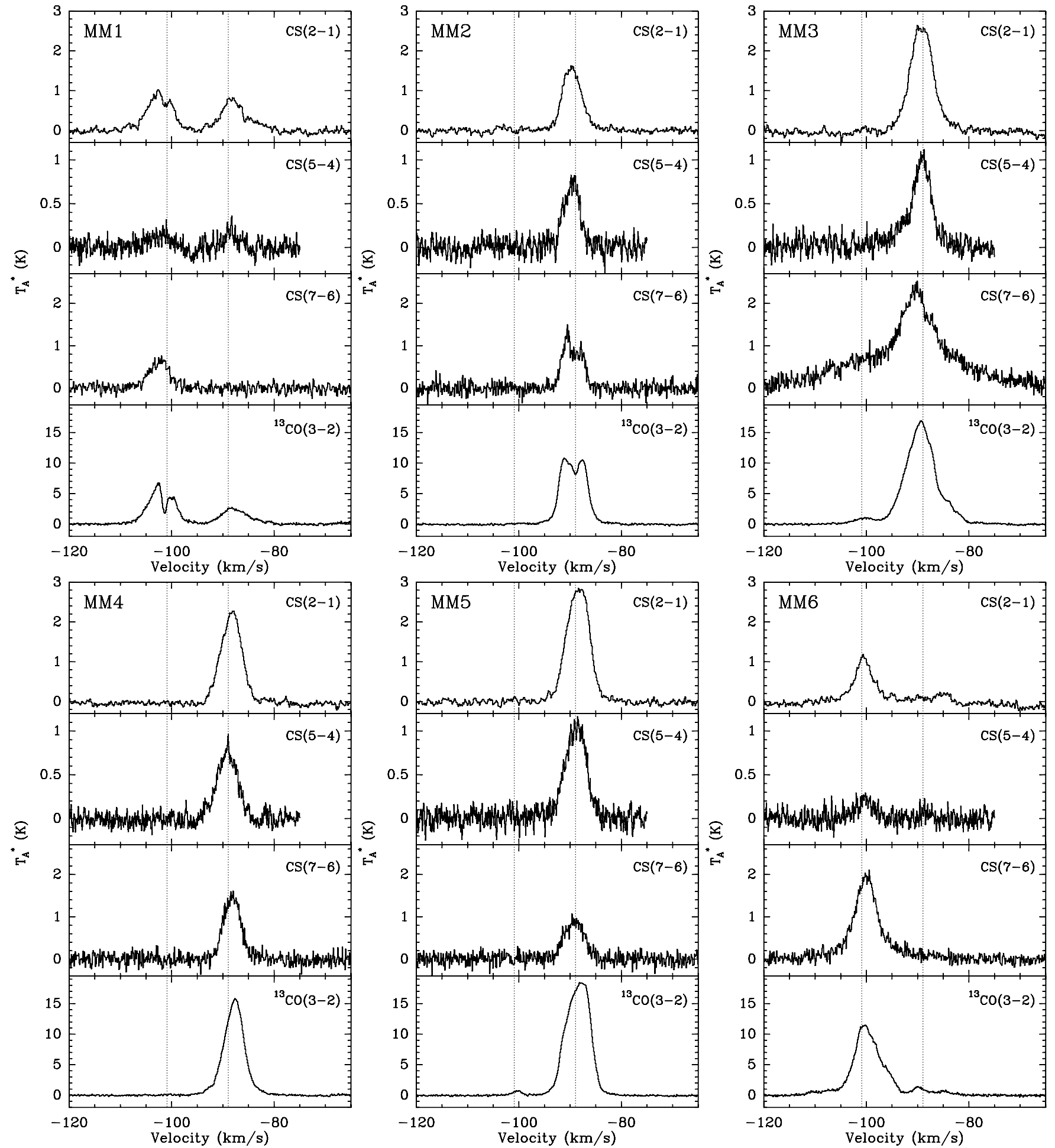

Figure 6. Spectra observed at the peak position of the millimeter clumps MM1 through MM6. The transition is given in the upper right corner. These spectra indicate that the emission arises from two different components: a high velocity component at $v=-88.9 \mathrm{~km} \mathrm{~s}^{-1}$, and a low velocity component at $v=-100.8 \mathrm{~km} \mathrm{~s}{ }^{-1}$, indicated with vertical dotted lines. Molecular sources associated with millimeter clumps MM2, MM3, MM4, and MM5 are part of a single structure at high velocity that we defined as the complex of clumps.

of component $\mathrm{B}$ and $\mathrm{C}$ are given in Column 9 of Table 7. Component $\mathrm{B}$ has a spectral index of the radio emission between 4.8 and $8.6 \mathrm{GHz}$ of $0.8 \pm 0.2$, similar the value expected for a spherical, isothermal, constant-velocity model of stellar wind (Reynolds 1986). The error in the determination of spectral indexes include the 5\% uncertainty in the absolute flux density scale. Component $\mathrm{C}$ spectral index has a value of $1.2 \pm 0.2$ already reported by Bronfman et al. (2008). This index suggests that source $\mathrm{C}$ corresponds to an ionized jet which is likely to drive the associated energetic molecular outflow.

Components A and D are more extended than B and C, and they present a decrease in the integrated flux density from 4.8 to $8.6 \mathrm{GHz}$. We attribute this particular behavior to an observational artifact that could be related with a less complete uv-coverage at higher frequency. An estimation of the flux density inside an area comparable with the beam size $(\sim 2$ '.5) gives an almost 


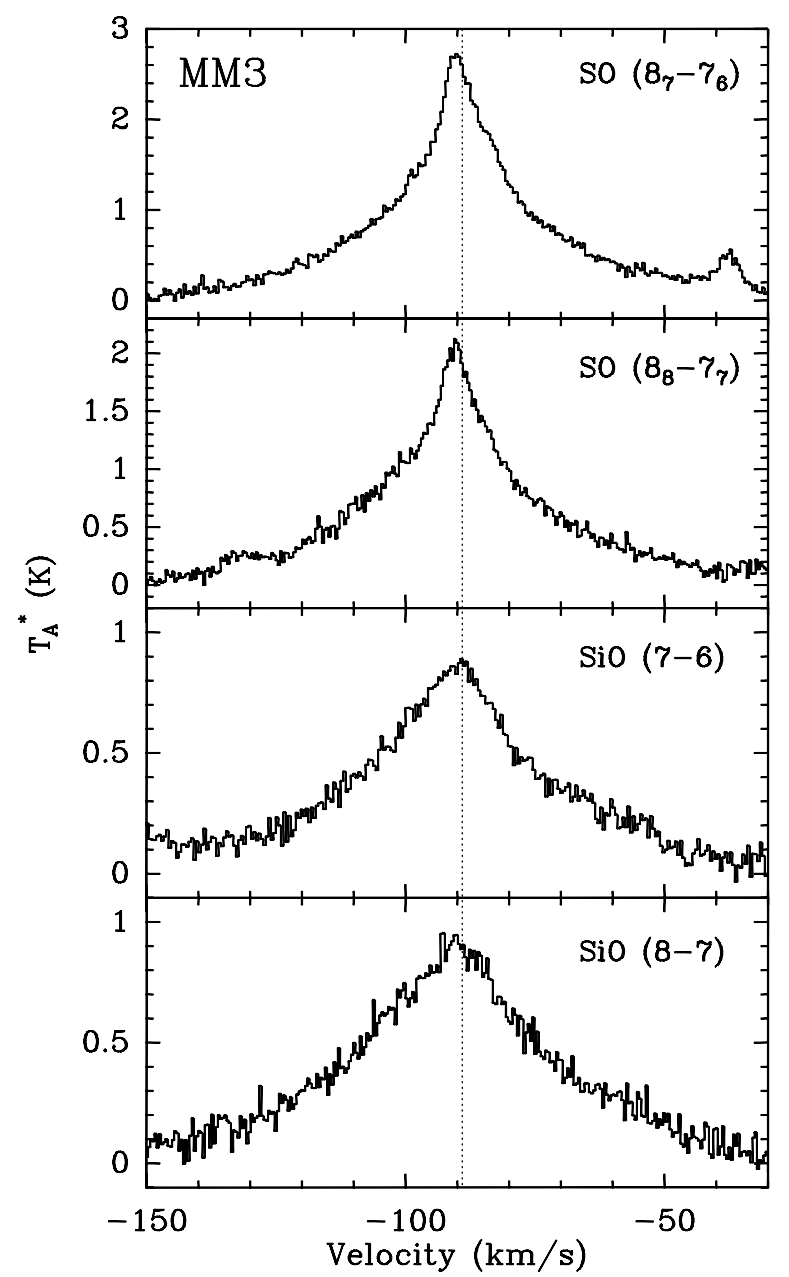

Figure 7. $\mathrm{SiO}$ and $\mathrm{SO}$ spectra observed toward the peak position of clump MM3, which is associated with the high-velocity molecular outflow G331.512-0.103. Transitions are given in the upper right corner. The vertical dotted line indicates the ambient gas velocity.

flat spectrum for component $\mathrm{A}$, and even though the flux still decreases with frequency for component $\mathrm{D}$, the flux difference was reduced from $47 \%$ to $30 \%$. We expected for both sources, A and $\mathrm{D}$, a flat spectral energy distribution in the radio continuum, consistent with the free-free emission from a thermal source.

\subsubsection{Maser Emission}

Earlier studies of masers across our Galaxy have shown that regions of high-mass star formation can be associated with $\mathrm{OH}, \mathrm{H}_{2} \mathrm{O}$, and Class II methanol masers (Caswell et al. 1995; Szymczak et al. 2002). In particular, $\mathrm{OH}$ masers are usually considered signposts of UCHII regions, while methanol masers are found associated to hot molecular cores, UCHII regions, and near-IR sources (Garay \& Lizano 1999; Bartkiewicz \& van Langevelde 2012). Therefore, $\mathrm{OH}$ and methanol masers often coincide, and they are found in regions with active star formation. With this in mind, we searched in methanol and $\mathrm{OH}$ surveys toward the Norma spiral arm region. We found three sources from the $6 \mathrm{GHz}$ methanol multibeam maser catalog from Caswell et al. (2011). Two of them, 331.5420.066 and 331.543-0.066, are toward the MM4 millimeter clump and they are coincident, within the errors, with the radio component $\mathrm{B}$. The velocity of the peak reported for these maser sources are $-86 \mathrm{~km} \mathrm{~s}^{-1}$ and $-84 \mathrm{~km} \mathrm{~s}^{-1}$, respectively, within $5 \mathrm{~km} \mathrm{~s}^{-1}$ difference from the molecular line velocities
Table 5

Sizes (FWHM) Obtained with $\mathrm{CS}(7 \rightarrow 6)$ Line

\begin{tabular}{lcccc}
\hline \hline Clump & $\begin{array}{c}\Delta v \\
\left(\mathrm{~km} \mathrm{~s}^{-1}\right)\end{array}$ & $\begin{array}{c}\text { Maj Axis } \\
\left({ }^{\prime \prime}\right)\end{array}$ & $\begin{array}{c}\text { Min Axis } \\
\left({ }^{\prime \prime}\right)\end{array}$ & $\begin{array}{c}\text { Radius } \\
(\mathrm{pc})\end{array}$ \\
\hline MM1 & $4.3 \pm 0.3$ & $35 \pm 3.6$ & $23 \pm 2.4$ & $0.30 \pm 0.03$ \\
MM2 & $4.4 \pm 0.1$ & $68 \pm 7.6$ & $47 \pm 5.3$ & $0.94 \pm 0.04$ \\
MM3 & $6.0 \pm 0.2$ & $37 \pm 1.4$ & $21 \pm 0.8$ & $0.29 \pm 0.01$ \\
MM4 & $4.6 \pm 0.1$ & $44 \pm 3.7$ & $24 \pm 1.9$ & $0.42 \pm 0.02$ \\
MM5 & $4.9 \pm 0.1$ & $110 \pm 17.3$ & $44 \pm 6.9$ & $1.19 \pm 0.07$ \\
MM6 & $6.3 \pm 0.1$ & $29 \pm 0.7$ & $25 \pm 0.6$ & $0.25 \pm 0.01$ \\
\hline
\end{tabular}

of MM4 shown in Table 4, and therefore consistent with the ambient velocity of the complex of clumps. The third $6668 \mathrm{MHz}$ methanol maser spot, 331.556-0.121 $\left(\alpha_{2000}=16^{\mathrm{h}} 12^{\mathrm{m}} 27^{\mathrm{s}} .21\right.$ and $\left.\delta_{2000}=-51^{\circ} 27^{\prime} 38^{\prime \prime} .2\right)$, is associated with the MM6 millimeter clump, it has a velocity at a peak emission of $-97 \mathrm{~km} \mathrm{~s}^{-1}$, similar to values found with molecular lines for MM6, and it is located $5^{\prime \prime} .8$ ( 3 times the beam size in the maser catalog) away from the peak position of radio component D. Each of these three maser spots has a counterpart OH maser (Caswell 1998, 2009). The peak position of radio component $\mathrm{C}\left(\alpha_{2000}=16^{\mathrm{h}} 12^{\mathrm{m}} 10^{\mathrm{s}} .04\right.$, $\left.\delta_{2000}=-51^{\circ} 28^{\prime} 37^{\prime \prime} .7\right)$ is coincident, within the errors, with the interferometrically derived position of an $1665 \mathrm{MHz}$ OH maser spot (G331.512-0.103; Caswell, 1998). No methanol class II emission was reported toward the outflow up to a detection limit of $0.6 \mathrm{Jy}$ (Caswell et al. 2011). The position of masers in the G331.5-0.1 GMC central region is shown in Figure 12, along their association with the radio continuum sources.

No methanol or $\mathrm{OH}$ emission is associated with the MM1, MM2, and MM5 clumps. While MM1 is associated with the radio component $\mathrm{A}$ and with an RMS source, no other signposts of star formation activity are found in MM2 and MM5, suggesting that these clumps are in a state prior to the formation of an UCHII region.

\section{DISCUSSION}

\subsection{Physical Properties of the GMC}

The mass of the G331.5-0.1 GMC can be derived from the CO NANTEN observations considering two approaches: (1) from the relation of the integrated intensity of $\mathrm{CO}$ line emission with $\mathrm{H}_{2}$ mass, and (2) using the local thermodynamic equilibrium (LTE) formalism.

In the first method, the column density $N\left(\mathrm{H}_{2}\right)$ of the GMC is considered proportional to the $\mathrm{CO}$ emission integrated in velocity $\left[I(\mathrm{CO})=\int T_{\mathrm{mb}}\left({ }^{12} \mathrm{CO}\right) d v\left(\mathrm{~K} \mathrm{~km} \mathrm{~s}^{-1}\right)\right]$,

$$
N\left(\mathrm{H}_{2}\right)=X(\mathrm{CO}) I(\mathrm{CO}) \text {, }
$$

where $X(\mathrm{CO})$ corresponds to the Galactic average $I(\mathrm{CO})$-to$N\left(\mathrm{H}_{2}\right)$ conversion factor. With this approach, the luminosity of $\mathrm{CO}, L_{\mathrm{CO}}=D^{2} \iint T_{\mathrm{mb}}\left({ }^{12} \mathrm{CO}\right) d v d \Omega$, is proportional to the total mass of the cloud, $M_{\mathrm{CO}}=\alpha_{\mathrm{CO}} L_{\mathrm{CO}}$. Here we assumed that $X(\mathrm{CO})=2.3 \times 10^{20} \mathrm{~cm}^{-2}\left(\mathrm{~K} \mathrm{~km} \mathrm{~s}^{-1}\right)^{-1}$ (Kennicutt \& Evans 2012), which implies $\alpha_{\mathrm{CO}}=4.6 M_{\odot}\left(\mathrm{K} \mathrm{km} \mathrm{s}^{-1} \mathrm{pc}^{2}\right)^{-1}$ with correction for helium.

From the $\mathrm{CO}$ emission integrated in the velocity range between -117.7 and $-73.3 \mathrm{~km} \mathrm{~s}^{-1}$, we measured a $\mathrm{CO}$ luminosity of $1.9 \times 10^{6}\left(\mathrm{~K} \mathrm{~km} \mathrm{~s}^{-1} \mathrm{pc}^{2}\right)$, giving a total mass of $8.7 \times 10^{6} M_{\odot}$ and an average column density of $3.2 \times 10^{22} \mathrm{~cm}^{-2}$.

We also estimate the column density and mass from the $\mathrm{C}^{18} \mathrm{O}$ observation following the derivation of physical parameters 

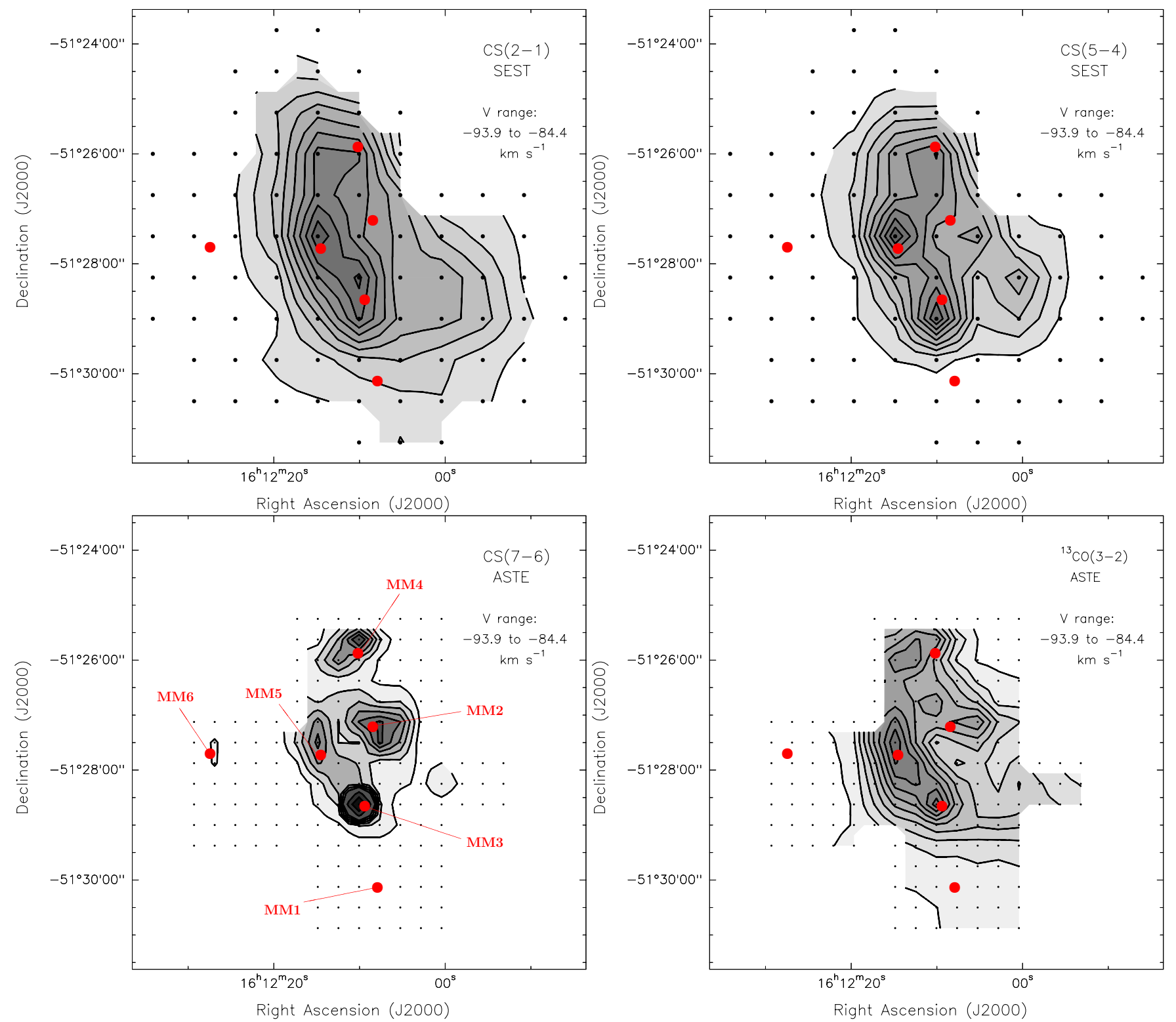

Figure 8. Contour maps of velocity-integrated emission, in the range from -93.9 to $-84.4 \mathrm{~km} \mathrm{~s}^{-1}$ from the central region of the G331.5-0.1 molecular cloud in four different transitions. For the $\operatorname{CS}(7 \rightarrow 6)$ map, the contour levels are drawn at $5 \%, 10 \%, 15 \%, 20 \%, 25 \%, 30 \%, 50 \%$, and $70 \%$ of the peak flux density $25.6 \mathrm{~K}$ km s ${ }^{-1}$. For $\mathrm{CS}(2 \rightarrow 1), \mathrm{CS}(5 \rightarrow 4)$ and ${ }^{13} \mathrm{CO}(3 \rightarrow 2)$ maps, the contours are drawn at $10 \%$ to $90 \%$, with interval of $10 \%$, of the peak intensity. For $\mathrm{CS}(2 \rightarrow 1)$, $\mathrm{CS}(5 \rightarrow 4)$ and ${ }^{13} \mathrm{CO}(3 \rightarrow 2)$, the peak intensities are $15.3,5.3$, and $161.7 \mathrm{~K} \mathrm{~km} \mathrm{~s}^{-1}$, respectively. The red points show the peak positions of the millimeter clumps MM1-MM6, as is labeled in the $\mathrm{CS}(7 \rightarrow 6)$ contour map. Molecular sources associated with millimeter clumps MM2, MM3, MM4, and MM5 are part of a single structure at high velocity that we defined as the complex of clumps.

(A color version of this figure is available in the online journal.)

Table 6

Fluxes from Continuum Observations

\begin{tabular}{|c|c|c|c|c|c|c|c|c|c|c|c|c|c|c|}
\hline \multirow[b]{2}{*}{ Region } & \multicolumn{2}{|c|}{ Millimeter } & \multicolumn{4}{|c|}{ IRAS Bands } & \multicolumn{4}{|c|}{ MSX Bands } & \multicolumn{4}{|c|}{ Spitzer Bands } \\
\hline & $\begin{array}{c}1.2 \mathrm{~mm} \\
(\mathrm{Jy})\end{array}$ & $\begin{array}{c}0.87 \mathrm{~mm} \\
\text { (Jy) }\end{array}$ & $\begin{array}{c}100 \mu \mathrm{m} \\
(\mathrm{Jy})\end{array}$ & $\begin{array}{c}60 \mu \mathrm{m} \\
(\mathrm{Jy})\end{array}$ & $\begin{array}{c}25 \mu \mathrm{m} \\
\text { (Jy) }\end{array}$ & $\begin{array}{c}12 \mu \mathrm{m} \\
(\mathrm{Jy})\end{array}$ & $\begin{array}{c}21.3 \mu \mathrm{m} \\
(\mathrm{Jy})\end{array}$ & $\begin{array}{c}14.7 \mu \mathrm{m} \\
(\mathrm{Jy})\end{array}$ & $\begin{array}{c}12.1 \mu \mathrm{m} \\
(\mathrm{Jy})\end{array}$ & $\begin{array}{c}8.3 \mu \mathrm{m} \\
(\mathrm{Jy})\end{array}$ & $\begin{array}{c}8 \mu \mathrm{m} \\
(\mathrm{Jy})\end{array}$ & $\begin{array}{c}5.8 \mu \mathrm{m} \\
(\mathrm{Jy})\end{array}$ & $\begin{array}{c}4.5 \mu \mathrm{m} \\
\text { (Jy) }\end{array}$ & $\begin{array}{c}3.6 \mu \mathrm{m} \\
(\mathrm{Jy})\end{array}$ \\
\hline Complex & 37.0 & 227.9 & 36517.0 & 30998.2 & 3855.8 & 649.1 & 2680.8 & 1143.3 & 1005.1 & 373.0 & 712.2 & 262.2 & 36.3 & 34.1 \\
\hline MM1 & 3.1 & 27.3 & 2254.0 & 629.3 & 24.9 & 3.7 & 22.9 & 13.1 & 21.4 & 13.1 & 25.0 & 7.5 & 0.7 & 0.9 \\
\hline MM2 & 6.4 & 38.3 & 8548.7 & 7948.4 & 1177.2 & 232.5 & 855.4 & 387.9 & 305.7 & 86.4 & 136.7 & 48.8 & 6.9 & 5.7 \\
\hline MM3 & 11.6 & 66.7 & 7687.6 & 6682.3 & 496.3 & 72.3 & 731.5 & 309.9 & 269.5 & 99.2 & 171.8 & 73.6 & 10.7 & 9.6 \\
\hline MM4 & 4.9 & 42.7 & 5098.2 & 5243.1 & 707.9 & 122.2 & 500.8 & 222.7 & 169.7 & 54.3 & 84.3 & 29.9 & 4.4 & 3.9 \\
\hline MM5 & 7.6 & 52.5 & 8618.1 & 6530.5 & 923.5 & 147.2 & 482.0 & 187.7 & 173.7 & 71.5 & 102.8 & 38.7 & 4.6 & 4.2 \\
\hline MM6 & 3.5 & 27.6 & 2086.1 & 1710.6 & 192.1 & 33.9 & 115.0 & 47.5 & 51.4 & 24.6 & 40.6 & 14.1 & 2.3 & 2.0 \\
\hline
\end{tabular}



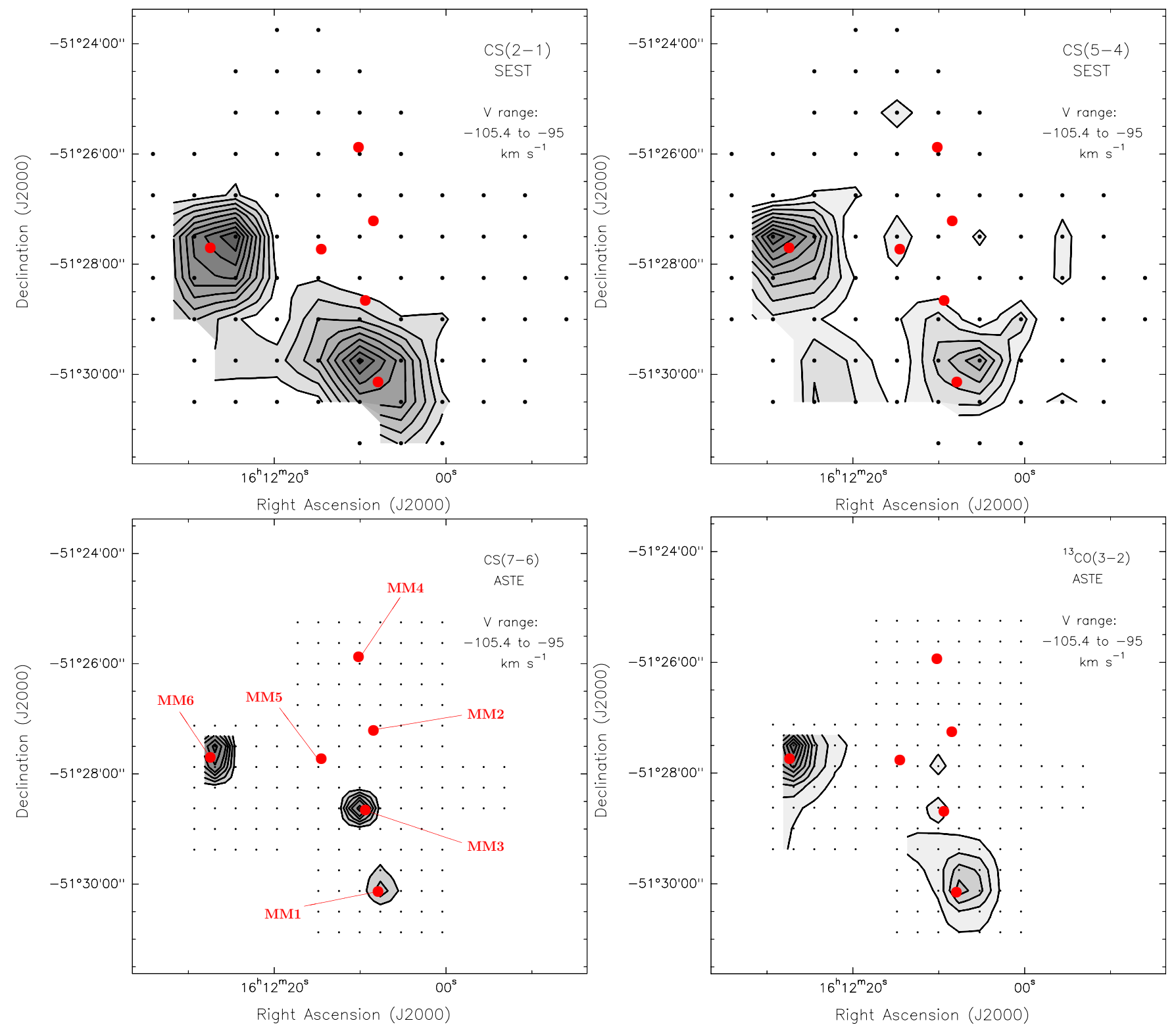

Figure 9. Contour maps of velocity-integrated emission, in the range between -105.4 and $-95 \mathrm{~km} \mathrm{~s}^{-1}$, from the central region of the G331.5-0.1 molecular cloud in four different transitions. For the $\operatorname{CS}(7 \rightarrow 6)$ map, the contour levels are drawn at $10 \%, 20 \%, 30 \%, 40 \%, 50 \%, 70 \%$, and $90 \%$ of the peak flux density $14.0 \mathrm{~K} \mathrm{~km} \mathrm{~s}{ }^{-1}$ For $\mathrm{CS}(2 \rightarrow 1), \mathrm{CS}(5 \rightarrow 4)$ and ${ }^{13} \mathrm{CO}(3 \rightarrow 2)$ maps, the contours are drawn at $10 \%$ to $90 \%$, with interval of $10 \%$, of the peak intensity. For $\mathrm{CS}(2 \rightarrow 1)$, the peak intensity is $5.2 \mathrm{~K} \mathrm{~km} \mathrm{~s}^{-1}$. For $\mathrm{CS}(5 \rightarrow 4)$, the peak intensity is $1.4 \mathrm{~K} \mathrm{~km} \mathrm{~s}^{-1}$. For ${ }^{13} \mathrm{CO}(3 \rightarrow 2)$, the peak intensity is $86.7 \mathrm{~K} \mathrm{~km} \mathrm{~s}{ }^{-1}$. The red points show the peak positions of the millimeter clumps MM1-MM6, as is labeled in the $\operatorname{CS}(7 \rightarrow 6)$ contour map. The emission observed in the $\operatorname{CS}(7 \rightarrow 6)$ map at $\alpha_{2000}=16^{\mathrm{h}} 12^{\mathrm{m}} 10^{\mathrm{s}} .13$ and $\delta_{2000}=-51^{\circ} 28^{\prime} 37^{\prime \prime} .5$ (toward the MM3 point) is related with the emission integration on the blue wing of the G331.512-0.103 outflow.

(A color version of this figure is available in the online journal.)

Table 7

Observed Parameters of Radio Sources Detected Toward G331.5-0.1

\begin{tabular}{|c|c|c|c|c|c|c|c|c|}
\hline Component & $\begin{array}{l}\text { Freq. } \\
(\mathrm{GHz})\end{array}$ & $\alpha(\mathrm{J} 2000)$ & $\delta(\mathrm{J} 2000)$ & $\begin{array}{c}\text { Flux Peak } \\
\left(\text { Jy beam }^{-1}\right)\end{array}$ & $\begin{array}{l}\text { Flux Density } \\
\text { (Jy) }\end{array}$ & $\begin{array}{l}\text { Beam } \\
(")\end{array}$ & $\begin{array}{l}\text { Deconv. Angular Size } \\
\left({ }^{\prime \prime}\right)\end{array}$ & Spectral Index \\
\hline \multirow[t]{2}{*}{ A } & 4.80 & 161207.510 & -513002.23 & 0.155 & 0.255 & $2.7 \times 1.8$ & $1.97 \times 1.54$ & $\ldots$ \\
\hline & 8.64 & 161207.471 & -513002.39 & 0.101 & 0.217 & $1.5 \times 1.0$ & $1.49 \times 1.34$ & \\
\hline \multirow[t]{2}{*}{$\overline{\mathrm{B}}$} & 4.80 & 161209.036 & -512547.83 & 0.125 & 0.141 & $2.7 \times 1.8$ & $0.81 \times 0.60$ & $0.8 \pm 0.2$ \\
\hline & 8.64 & 161209.038 & -512547.79 & 0.166 & 0.222 & $1.5 \times 1.0$ & $0.84 \times 0.36$ & \\
\hline \multirow[t]{2}{*}{$\mathrm{C}$} & 4.80 & 161210.039 & -512837.72 & 0.085 & 0.095 & $2.7 \times 1.8$ & $0.86 \times 0.37$ & $1.2 \pm 0.2$ \\
\hline & 8.64 & 161210.030 & -512837.66 & 0.158 & 0.194 & $1.5 \times 1.0$ & $0.61 \times 0.40$ & \\
\hline \multirow[t]{2}{*}{$\bar{D}$} & 4.80 & 161227.308 & -512732.54 & 0.066 & 0.135 & $2.7 \times 1.8$ & $2.30 \times 2.06$ & \\
\hline & 8.64 & 161227.264 & -512732.81 & 0.022 & 0.092 & $1.5 \times 1.0$ & $2.15 \times 1.87$ & \\
\hline
\end{tabular}




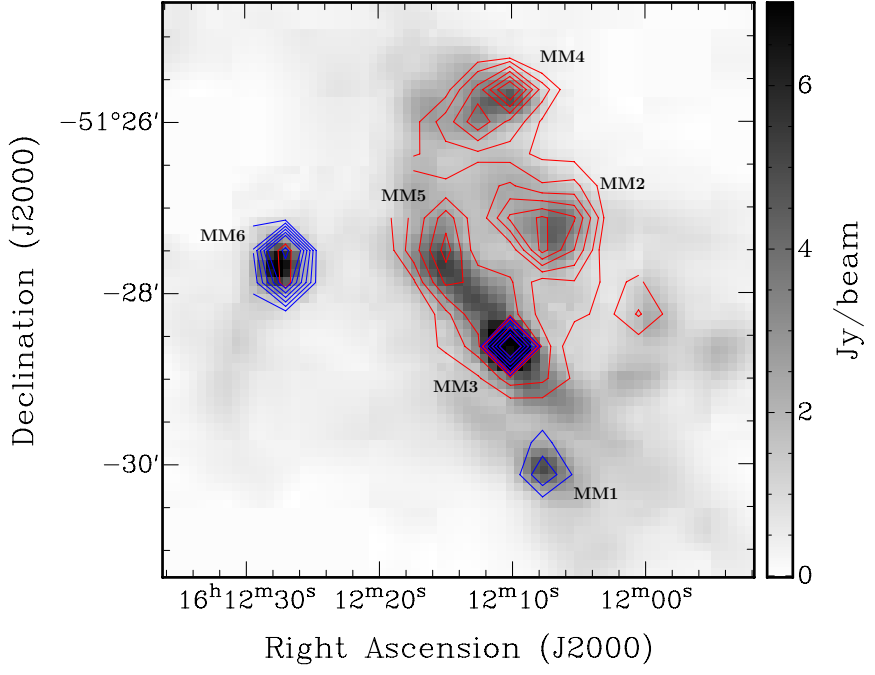

Figure 10. $0.87 \mathrm{~mm}$ grayscale image of the central region of the G331.5-0.1 GMC, overlaid with CS velocity integrated contour map. The contour levels are drawn at $5 \%, 10 \%, 15 \%, 20 \%, 25 \%, 30 \%, 50 \%$, and $70 \%$ of the peak flux density $25.6 \mathrm{~K} \mathrm{~km} \mathrm{~s}^{-1}$. The red contours show the integrated emission in the high velocity component of the spectra, integrated between -93.9 and $-84.4 \mathrm{~km} \mathrm{~s}^{-1}$. The blue contours show the low velocity component, integrated between -105.4 and $-95 \mathrm{~km} \mathrm{~s}^{-1}$. Labels MM1-MM6 show the associated millimeter clump for each CS source. Molecular sources associated with millimeter clumps MM2, MM3, MM4, and MM5 are part of a single structure at high velocity that we defined as the complex of clumps. presented by Bourke et al. (1997). Assuming that the emission is optically thin,

$$
\begin{aligned}
N\left(\mathrm{C}^{18} \mathrm{O}\right)= & 2.42 \times 10^{14} \frac{T_{\mathrm{ex}}+0.88}{1-\exp \left(-5.27 / T_{\mathrm{ex}}\right)} \\
& \times \frac{1}{J\left(T_{\mathrm{ex}}\right)-J\left(T_{\mathrm{bg}}\right)} \int T_{\mathrm{mb}}\left(\mathrm{C}^{18} \mathrm{O}\right) d v,
\end{aligned}
$$

and

$$
\begin{aligned}
\left(\frac{M_{\mathrm{LTE}}}{M_{\odot}}\right)= & 1.44\left(\frac{\mu_{m}}{2.72 m_{\mathrm{H}}}\right)\left(\frac{\left[\mathrm{H}_{2} / \mathrm{C}^{18} \mathrm{O}\right]}{3.3 \times 10^{6}}\right)\left(\frac{D}{\mathrm{kpc}}\right)^{2} \\
& \times \frac{T_{\mathrm{ex}}+0.88}{1-\exp \left(-5.27 / T_{\mathrm{ex}}\right)} \frac{1}{J\left(T_{\mathrm{ex}}\right)-J\left(T_{\mathrm{bg}}\right)} \\
& \times \iint T_{\mathrm{mb}}\left(\mathrm{C}^{18} \mathrm{O}\right) d v d \Omega
\end{aligned}
$$

where $T_{\mathrm{ex}}$ is the excitation temperature of the transition, $T_{\mathrm{bg}}$ is the background temperature, $v$ is in $\mathrm{km} \mathrm{s}^{-1}, \Omega$ is in $\operatorname{arcmin}^{2}$, and

$$
J(T)=\frac{h v}{k} \frac{1}{\exp (h v / k T)-1}
$$

We assumed an abundance ratio $\left[\mathrm{CO} / \mathrm{C}^{18} \mathrm{O}\right]=330$, which is the suggested value for GMCs inside the $4 \mathrm{kpc}$ Galactic molecular ring (Wilson \& Rood 1994). Considering an average

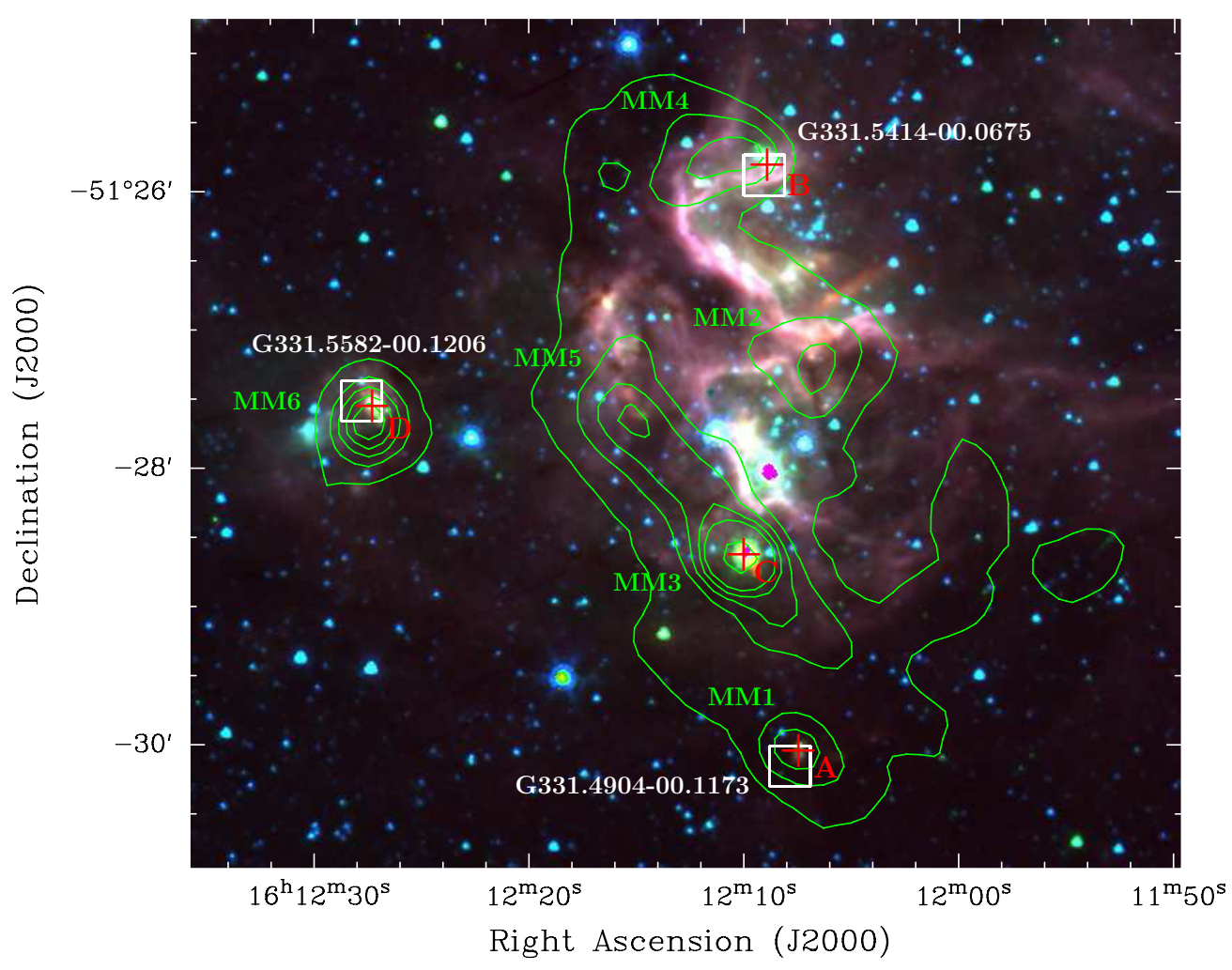

Figure 11. Contour map of the $0.87 \mathrm{~mm}$ continuum emission from the G331.5-0.1 region overlaid on a three color image of the mid infrared emission, made using the IRAC filters $1(3.6 \mu \mathrm{m}$, blue), $2(4.5 \mu \mathrm{m}$, green), and $4(8 \mu \mathrm{m}$, red). Contour levels are drawn at $10 \%, 20 \%, 30 \%, 40 \%$, 50\%, and $90 \%$ of the peak flux density $13.8 \mathrm{Jy}_{\text {beam }}^{-1}$. Millimeter sources are labeled in the map with MM1, MM2, MM3, MM4, MM5, and MM6. Red crosses mark the positions of the four radio emission components in the central region of the G331.5-0.1 GMC (A, B, C and D). Component B and D are associated with methanol and OH masers. Component $\mathrm{C}$ is associated only with $\mathrm{OH}$ maser emission. White boxes mark the position of the three RMS sources in the region (G331.4904-00.1173, G331.5414-00.0675, and G331.5582-00.1206). The RMS box sizes are 18" to account for the beam of MSX observation. 


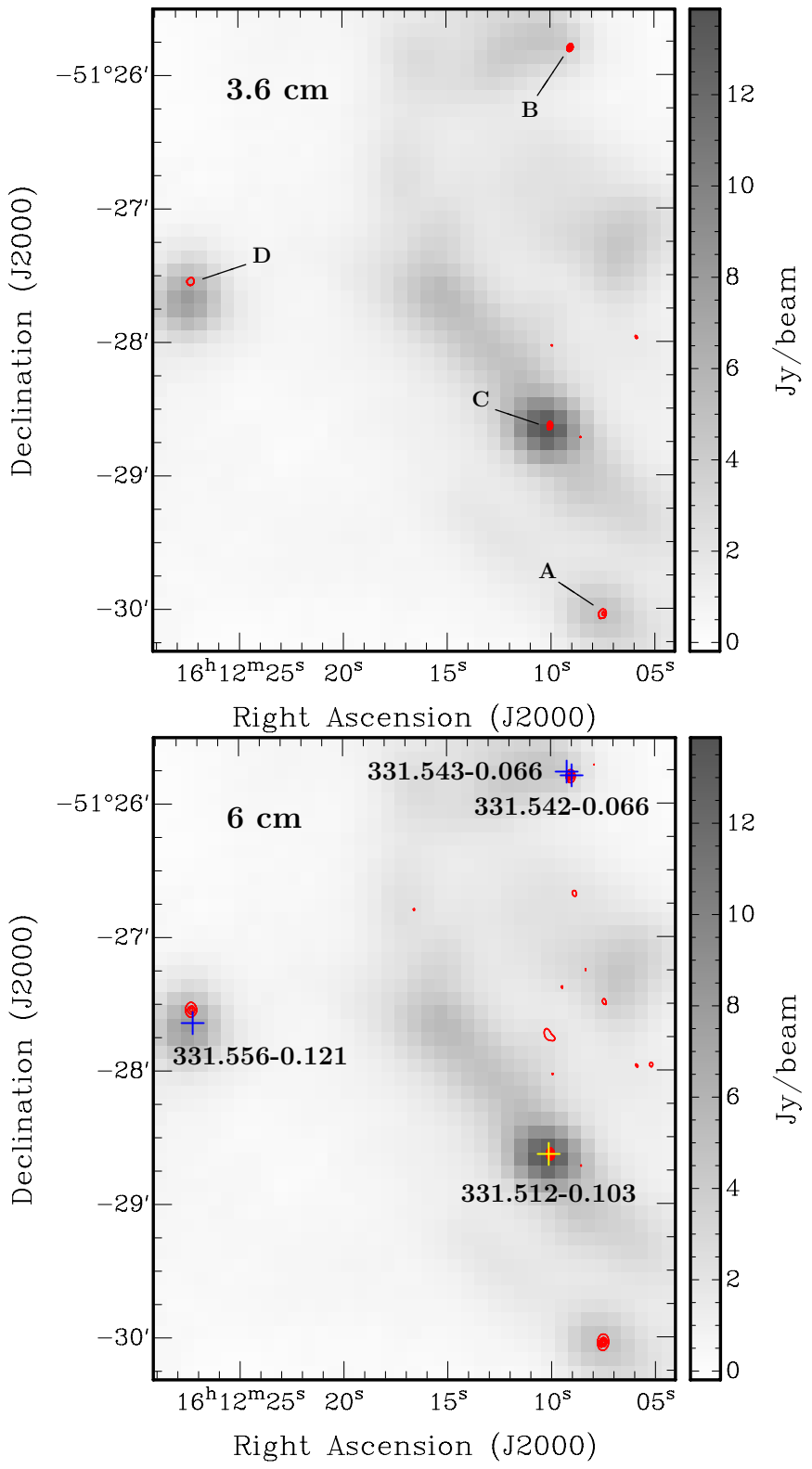

Figure 12. Contour maps of the radio emission from the G331.5-0.1 GMC central region at $3.6 \mathrm{~cm}$ (top) and $6 \mathrm{~cm}$ (bottom), overlaid on $0.87 \mathrm{~mm}$ continuum emission. The contours are drawn at $10 \%, 30 \%, 60 \%$, and $90 \%$ of the peak flux. For the $6 \mathrm{~cm}$ map, the peak intensity is $153.0 \mathrm{mJy} \mathrm{beam}^{-1}, \mathrm{rms}$ noise is $1.04 \mathrm{mJy}$ beam $^{-1}$. For the $3.6 \mathrm{~cm}$ map, the peak intensity is $159.5 \mathrm{mJy}$ beam $^{-1}$, rms noise is $1.09 \mathrm{mJy}$ beam $^{-1}$. The blue crosses in the $6 \mathrm{~cm}$ map show the position of methanol masers in the central region of the G331.5-0.1 GMC. All methanol masers here are associated with $\mathrm{OH}$ masers. The yellow cross shows the position of the $\mathrm{OH}$ maser, without methanol counterpart, toward the G331.512-0.103 outflow.

(A color version of this figure is available in the online journal.)

temperature of $15 \mathrm{~K}$, the total mass value for this region is then $3.5 \times 10^{6} M_{\odot}$, with an average column density $N\left(\mathrm{C}^{18} \mathrm{O}\right)=$ $4.0 \times 10^{15} \mathrm{~cm}^{-2}$. The mass estimated is less than half that the mass obtained from $\mathrm{CO}$, but the denser material traced by $\mathrm{C}^{18} \mathrm{O}$ is emitting in a smaller area. Since the continuum millimeter sources are seen in the $\mathrm{C}^{18} \mathrm{O}$ map concentrated within the area defined for the G331.5-0.1 central region, we estimated the column density toward the position of the peak emission on this map, considering a higher temperature than the average value used for the GMC. For $T=20 \mathrm{~K}$, we obtained $N\left(\mathrm{C}^{18} \mathrm{O}\right)=2.6 \times 10^{16} \mathrm{~cm}^{-2}$.
Table 8

Spectral Energy Distributions

\begin{tabular}{lccccccccccccc}
\hline \hline Region & $\begin{array}{c}T^{\mathrm{c}} \\
(\mathrm{K})\end{array}$ & $\beta^{\mathrm{c}}$ & $\begin{array}{c}v_{0}{ }^{\mathrm{c}} \\
(\mathrm{THz})\end{array}$ & $\begin{array}{c}\theta^{\mathrm{c}} \\
\left.{ }^{\prime \prime}\right)\end{array}$ & $\begin{array}{c}T^{\mathrm{w}} \\
(\mathrm{K})\end{array}$ & $\beta^{\mathrm{w}}$ & $\begin{array}{r}v_{0}{ }^{\mathrm{w}} \\
(\mathrm{THz})\end{array}$ & $\begin{array}{c}\theta^{\mathrm{w}} \\
\left.{ }^{\prime \prime}\right)\end{array}$ & $\begin{array}{c}T^{\mathrm{h}} \\
(\mathrm{K})\end{array}$ & $\beta^{\mathrm{h}}$ & $\begin{array}{c}v_{0}{ }^{\mathrm{h}} \\
(\mathrm{THz})\end{array}$ & $\begin{array}{c}\theta^{\mathrm{h}} \\
\left({ }^{\prime \prime}\right)\end{array}$ \\
\hline Complex & 30 & 2.2 & 7.3 & 200 & 114 & 1.0 & 25 & 4.3 & 302 & 1.0 & 316 & 0.85 \\
MM1 & 29 & 1.7 & 6.1 & 33 & $\ldots$ & $\ldots$ & $\ldots$ & $\ldots$ & $\ldots$ & $\ldots$ & $\ldots$ & $\ldots$ \\
MM2 & 34 & 1.9 & 5.8 & 50 & 136 & 1.0 & 26 & 1.8 & $\ldots$ & $\ldots$ & $\ldots$ & $\ldots$ \\
MM3 & 35 & 1.6 & 5.9 & 40 & 109 & 1.0 & 29 & 2.0 & $\ldots$ & $\ldots$ & $\ldots$ & $\ldots$ \\
MM4 & 30 & 1.9 & 5.8 & 50 & 112 & 1.0 & 24 & 2.3 & $\ldots$ & $\ldots$ & $\ldots$ & $\ldots$ \\
MM5 & 33 & 1.8 & 5.7 & 51 & 114 & 1.0 & 28 & 2.4 & $\ldots$ & $\ldots$ & $\ldots$ & $\ldots$ \\
MM6 & 31 & 1.7 & 5.8 & 30 & 112 & 1.0 & 28 & 1.2 & $\ldots$ & $\ldots$ & $\ldots$ & $\ldots$ \\
\hline
\end{tabular}

Notes.

c Cold component.

w Warm component.

${ }^{\mathrm{h}}$ Hot component.

\subsection{Physical Properties of the G331.5-0.1 Central Region}

\subsubsection{Spectral Energy Distributions}

To determine the dust temperature and bolometric luminosity of the GMC central region and each clump, we fit the SED using a modified blackbody model,

$$
S_{v}=\Omega B_{v}\left(T_{d}\right)\left\{1-\exp \left(-\tau_{v}\right)\right\}
$$

where $S_{v}$ is the flux density of the source, $B_{v}$ is the Planck function, $\Omega$ is the solid angle subtended by the emitting region, and $T_{d}$ is the dust temperature. The optical depth, $\tau_{\nu}$, is assumed to depend on frequencies, $\tau_{v}=\left(v / \nu_{0}\right)^{\beta}$, with $\beta$ the power law spectral index and $v_{0}$ the frequency at which the optical depth is unity.

SEDs were fit for each clump and for the region harboring the complex of clumps at high velocity. The flux densities were measured in the areas shown in dashed boxes in Figure 5, using the data observed at $1.2 \mathrm{~mm}$ and $0.87 \mathrm{~mm}$, and at four IRAS, MSX, and Spitzer bands (see Table 6). A good fit to the emission from the complex of clumps required three dust components at different temperatures (see Figure 14). For the individual clumps, we only considered a two-component model, without considering the Spitzer data (Figure 15). In the case of the MM1 clump, the source is only marginally detected at short wavelengths, and therefore we only considered a cold component for the model. The parameters obtained (see Table 8) are typical of regions associated with high-mass star formation (Faúndez et al. 2004). For the six clumps in the G331.5-0.1 central region, the average temperature of the cold fit component is $32 \mathrm{~K}$, and for the region containing the complex of clumps, the cold component has a fitted temperature of $30 \mathrm{~K}$. The average value of the spectral indexes of the cold component for the six clumps is 1.8 , which is consistent with the OH5 model from Ossenkopf \& Henning (1994). The steeper value of the spectral index found for the region containing the complex of clumps at high velocity could be due to low recovery of some extended flux in the $1.2 \mathrm{~mm}$ image. The bolometric luminosities were obtained according to:

$$
L_{\text {bol }}=F_{\text {int }} 4 \pi d^{2},
$$

with $d$ the distance of the source. The results are tabulated in Table 9. The clumps have an average bolometric luminosity of $L_{\text {bol }}=5.7 \times 10^{5} L_{\odot}$, above the average value derived by Faúndez et al. (2004) of $2.3 \times 10^{5} L_{\odot}$. If the source of energy of each clump is due to a single object, then it will correspond to a star with spectral type of O6 ZAMS or earlier (Panagia 


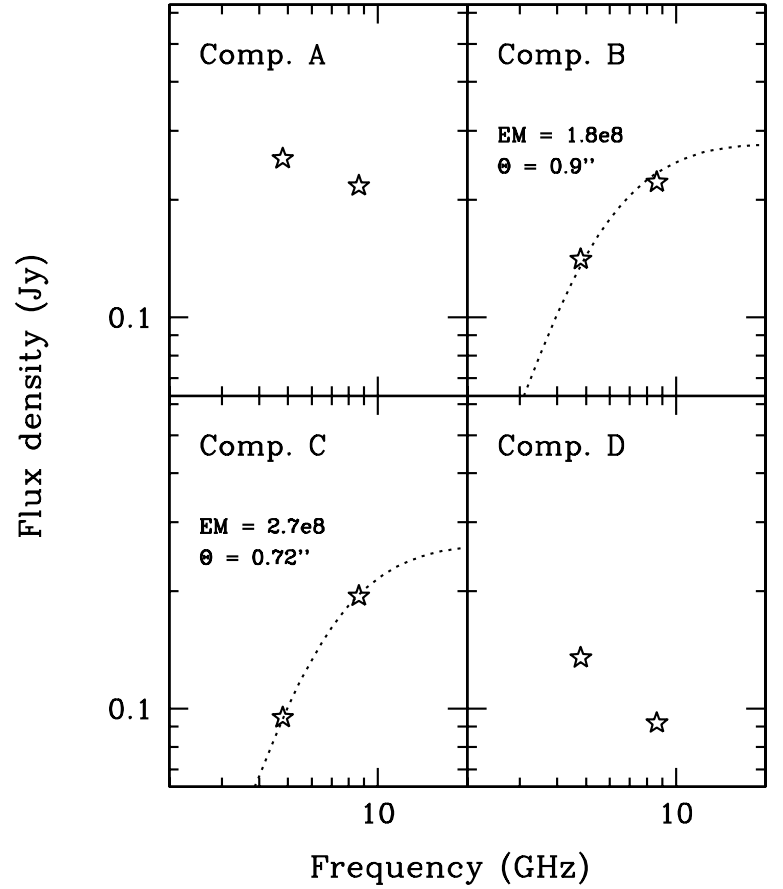

Figure 13. Spectral energy distributions at short frequency for each radio component found in the central region of the G331.5-0.1 GMC. For components $\mathrm{B}$ and $\mathrm{C}$, the dotted line is a fit of the spectrum using modified blackbody function of the form $B_{v}\left(T_{e}\right)\left[1-\exp \left(-\tau_{v}\right)\right]$, with $\tau_{v} \propto T_{e}^{-1.35} v^{-2.1} \mathrm{EM}$. The emission measure EM is in units of $\left[\mathrm{pc} \mathrm{cm}^{-6}\right]$. The $T_{e}$ considered is $10000 \mathrm{~K}$. The spectral index of components B and C are $0.8 \pm 0.2$ and $1.2 \pm 0.2$, respectively.

Table 9

Bolometric Luminosities of Complex and Individual Clumps

\begin{tabular}{lcc}
\hline \hline Region & $\begin{array}{c}\text { Integrated Flux } \\
\left(10^{6} \mathrm{Jy} \mathrm{GHz}\right)\end{array}$ & $\begin{array}{c}\text { Bolometric Luminosity } \\
\left(10^{6} L_{\odot}\right)\end{array}$ \\
\hline Complex & 252.2 & 4.4 \\
MM1 & 7.2 & 0.1 \\
MM2 & 57.6 & 1.0 \\
MM3 & 40.9 & 0.7 \\
MM4 & 35.5 & 0.6 \\
MM5 & 48.2 & 0.8 \\
MM6 & 12.3 & 0.2 \\
\hline
\end{tabular}

1973). However, an inspection of Figure 11 shows that the clumps are unlikely to be associated with just one star. Most likely the G331.5-0.1 central region has several early type stars, considering, for example, that a dozen of O9 ZAMS stars will produce the luminosity of a single O6 ZAMS star.

\subsubsection{Masses and Densities}

Using the millimeter continuum observations, we determined the mass of each clump from the expression:

$$
M_{d}=\frac{S_{v} D^{2}}{k_{v} B_{v}\left(T_{d}\right)}
$$

where $S_{\nu}$ is the flux density, $D$ is the distance to the source, $k_{\nu}$ is the dust mass coefficient, $B_{v}$ is the Planck function, and $T_{d}$ is the dust temperature.

We use a dust opacity of $1 \mathrm{~cm}^{2} \mathrm{~g}^{-1}$ at $1.2 \mathrm{~mm}$ and $1.89 \mathrm{~cm}^{2} \mathrm{~g}^{-1}$ at $0.87 \mathrm{~mm}$, which are values computed for typical conditions of dense protostellar objects (Ossenkopf \& Henning 1994). The opacity at $0.87 \mathrm{~mm}$ is obtained from a linear interpolation in the OH5 model of grains with thin ice mantles. Assuming a

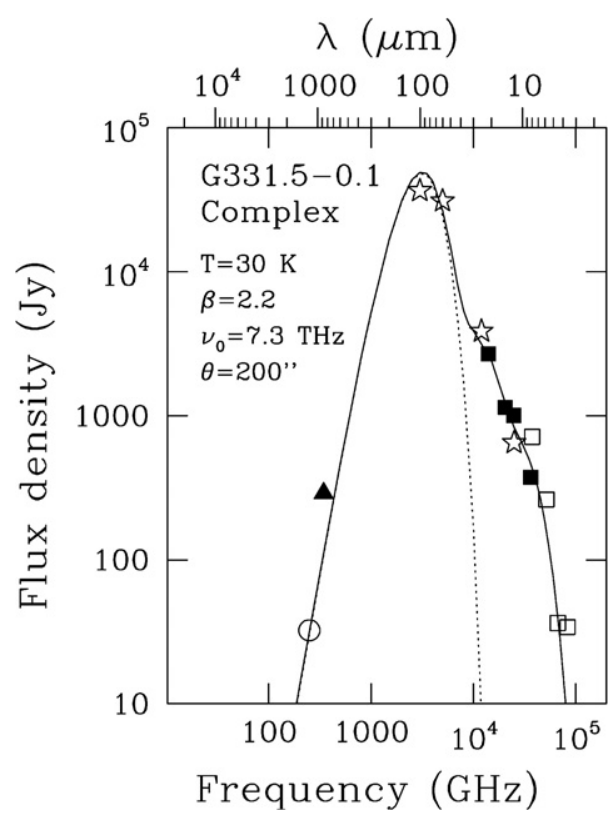

Figure 14. Spectral energy distribution of the region harboring the G331.50.1 complex of clumps. From left to right, the circle marks the SIMBA flux, the triangle the LABOCA flux, the stars mark IRAS fluxes, filledsquares MSX fluxes, and open-squares Spitzer fluxes. The solid curve is a fit to the spectrum using three modified blackbody functions of the form $B_{v}\left(T_{e}\right)\left[1-\exp \left(-\left(v / \nu_{0}\right)^{\beta}\right)\right]$, with different temperatures (cold, warm and hot components). The fitted parameters of the cold component (represented as the dotted line) are listed.

Table 10

Masses and Densities Derived for the Clumps

\begin{tabular}{|c|c|c|c|c|c|}
\hline \multirow[b]{2}{*}{ Clump } & \multirow{2}{*}{$\begin{array}{c}0.87 \mathrm{~mm} \\
\text { Mass } \\
\left(10^{3} M_{\odot}\right)\end{array}$} & \multicolumn{3}{|c|}{$1.2 \mathrm{~mm}$} & \multirow{2}{*}{$\begin{array}{c}\mathrm{CS}(7 \rightarrow 6) \\
\text { Virial Mass } \\
\left(10^{3} M_{\odot}\right)\end{array}$} \\
\hline & & $\begin{array}{c}\text { Mass } \\
\left(10^{3} M_{\odot}\right)\end{array}$ & $\begin{array}{c}\text { Density } \\
\left(10^{4} \mathrm{~cm}^{-3}\right)\end{array}$ & $\begin{array}{l}\text { Surface Density } \\
\quad\left(\mathrm{g} \mathrm{cm}^{-2}\right)\end{array}$ & \\
\hline MM1 & 5.1 & 1.9 & 2.9 & 0.30 & $1.2 \pm 0.2$ \\
\hline MM2 & 5.8 & 3.2 & 1.6 & 0.24 & $3.8 \pm 0.3$ \\
\hline MM3 & 9.7 & 5.6 & 10.9 & 1.03 & $2.2 \pm 0.2$ \\
\hline MM4 & 7.6 & 2.8 & 1.4 & 0.21 & $1.9 \pm 0.1$ \\
\hline MM5 & 8.2 & 3.9 & 1.9 & 0.29 & $6.0 \pm 0.4$ \\
\hline MM6 & 4.7 & 1.9 & 3.7 & 0.35 & $2.1 \pm 0.1$ \\
\hline
\end{tabular}

dust-to-gas mass ratio of $R=M_{d} / M_{g}=0.01$, the total mass of each clump is given by:

$$
\begin{aligned}
\left(\frac{M_{1.2 \mathrm{~mm}}}{M_{\odot}}\right)= & 20.82\left(\frac{S}{\mathrm{Jy}}\right)\left(\frac{1 \mathrm{~cm}^{2} \mathrm{~g}^{-1}}{\kappa_{1.2 \mathrm{~mm}}}\right)\left(\frac{D}{\mathrm{kpc}}\right)^{2} \\
& \times\left\{\exp \left(\frac{11.990}{T_{d}}\right)-1\right\} \\
\left(\frac{M_{0.87 \mathrm{~mm}}}{M_{\odot}}\right)= & 4.29\left(\frac{S}{\mathrm{Jy}}\right)\left(\frac{1.89 \mathrm{~cm}^{2} \mathrm{~g}^{-1}}{\kappa_{0.87 \mathrm{~mm}}}\right)\left(\frac{D}{\mathrm{kpc}}\right)^{2} \\
& \times\left\{\exp \left(\frac{16.538}{T_{d}}\right)-1\right\} .
\end{aligned}
$$

The mass of the clumps is estimated using the temperature obtained from the SED fitting. The masses estimated from the emission at $0.87 \mathrm{~mm}$ and $1.2 \mathrm{~mm}$ are given in Table 10 . If we instead considered a fiducial temperature $T=20$, for example, the masses will be in general larger by a factor of 


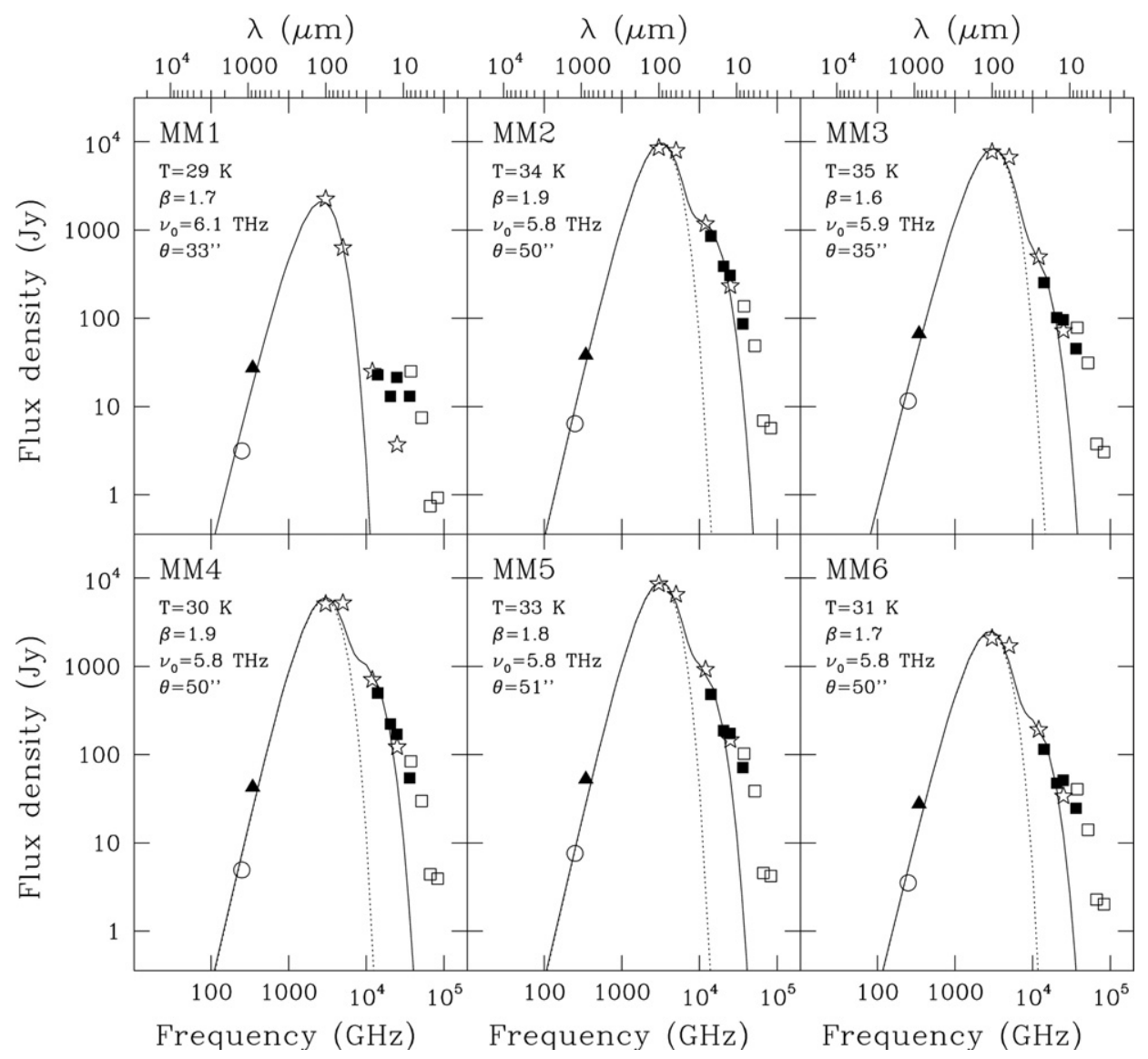

Figure 15. Spectral energy distributions of each millimeter clump in the G331.5-0.1 central region. The symbols are the same as in Figure 14. The solid curve is a fit to the spectrum using two modified blackbody functions of the form $B_{v}\left(T_{e}\right)\left[1-\exp \left(-\left(v / v_{0}\right)^{\beta}\right)\right]$, with different temperatures (cold and warm components). In the case of MM1, only one modified blackbody function was considered. The fitted parameters of the cold component (represented as the dotted line) are listed on the upper left.

two with respect to the masses estimated using the temperature from SED fitting. We note that the masses obtained from $1.2 \mathrm{~mm}$ are lower than the masses obtained from the $0.87 \mathrm{~mm}$ observations typically by a factor of two. Uncertainties in the dust opacities or in the dust temperatures could give other possible explanations. A better determination of the power law index $\beta$ of the dust optical depth could improve this value. Considering the results from the $1.2 \mathrm{~mm}$ observations, the average mass of the millimeter clumps in the central region is $3.2 \times 10^{3} M_{\odot}$. The total mass and size of the complex of clumps are $\sim 2.1 \times 10^{4} M_{\odot}$ and $\sim 15 \mathrm{pc}$. These values are bigger than the average masses and sizes of dust compact sources associated with high-mass star-forming regions $\left(\sim 5 \times 10^{3} M_{\odot}\right.$ and $\sim 1 \mathrm{pc}$; Faúndez et al. 2004). However, each clump is by itself like one of these sources.

Columns 4 and 5 in Table 10 give the number density and surface density estimated from the emission at $1.2 \mathrm{~mm}$ for each clump. The density and the average surface density were computed using the expressions

$$
n=\frac{M}{(4 / 3) \pi R^{3} \mu m_{H}} ; \quad \sum=\frac{M}{\pi R^{2}},
$$

assuming spherical morphologies and a mean mass per particle of $\mu=2.29$ (corrected for helium). The clumps have an average gas surface density of $0.4 \mathrm{~g} \mathrm{~cm}^{-2}\left(\sim 1900 M_{\odot} \mathrm{pc}^{-2}\right)$. The values of the surface densities are similar to those of the densest clumps reported in a study of massive star-forming regions by Dunham et al. (2011), and they are above the surface density threshold of $\Sigma_{\mathrm{th}}=129 \pm 14 M_{\odot} \mathrm{pc}^{-2}$ found by Heiderman et al. (2010) for "efficient" star formation. In an independent study, Lada et al. (2010) found a threshold of $\Sigma_{\text {th }}=116 M_{\odot} \mathrm{pc}^{-2}$. Only one of the millimeter clumps in the G331.5-0.1 central region have gas surface densities above $1 \mathrm{~g} \mathrm{~cm}^{-2}$, which is the theoretical lower limit determined by Krumholz \& McKee (2008) to avoid excessive fragmentation and form massive stars. Nevertheless, the inner and densest parts of each clump, mapped with a high-density gas tracer such as $\operatorname{CS}(7 \rightarrow 6)$, will exceed this requirement.

\subsubsection{Virial and LTE Masses}

For a spherical, nonrotating cloud of radius $\mathrm{R}$, and a Gaussian velocity profile $\Delta v$ (FWHM), the virial mass is given by

$$
\left(\frac{M_{\text {virial }}}{M_{\odot}}\right)=210\left(\frac{\Delta v}{\mathrm{~km} \mathrm{~s}^{-1}}\right)^{2}\left(\frac{R}{\mathrm{pc}}\right),
$$

assuming that the broadening due to optical depth is negligible. Column 6 in Table 10 shows the virial mass obtained for each clump, determined from the $\mathrm{CS}(7 \rightarrow 6)$ molecular line. The radius of the clumps was computed from the deconvolved angular size measured from the maps, and $\Delta v$ from the spatially average CS line emission (see Table 5). In the case of MM3, the outflow clump, we considered only the Gaussian component corresponding to the ambient gas emission. The total mass obtained for the complex of clumps is $1.4 \times 10^{4} \mathrm{M}_{\odot}$. 
We also estimate the mass of the complex of clumps from the ${ }^{13} \mathrm{CO}(3 \rightarrow 2)$ observations using the LTE formalism, assuming that this transition is optically thin. Using Equation (A4) from Bourke et al. (1997), the mass is estimated from the ${ }^{13} \mathrm{CO}$ line in a similar way than the $\mathrm{C}^{18} \mathrm{O}$ mass presented in Section 4.1:

$$
\begin{aligned}
\left(\frac{M_{\mathrm{LTE}}}{M_{\odot}}\right)= & 0.082\left(\frac{\mu_{m}}{2.72 m_{\mathrm{H}}}\right)\left(\frac{\left[\mathrm{H}_{2} /{ }^{13} \mathrm{CO}\right]}{5.3 \times 10^{5}}\right)\left(\frac{D}{\mathrm{kpc}}\right)^{2} \\
& \times \frac{T_{\mathrm{ex}}+0.88}{1-\exp \left(-15.87 / T_{\mathrm{ex}}\right)} \frac{\exp \left(15.87 / T_{\mathrm{ex}}\right)}{J\left(T_{\mathrm{ex}}\right)-J\left(T_{\mathrm{bg}}\right)} \\
& \times \iint T_{\mathrm{mb}}\left({ }^{13} \mathrm{CO}\right) d v d \Omega
\end{aligned}
$$

where $v$ is in $\mathrm{km} \mathrm{s}^{-1}$ and $\Omega$ is in $\operatorname{arcmin}^{2}$. We considered an excitation temperature of $30 \mathrm{~K}$ and a $\left[\mathrm{CO} /{ }^{13} \mathrm{CO}\right]$ abundance ratio of 53 (Wilson \& Rood 1994). The mass obtained for the complex of clumps is $2.9 \times 10^{4} M_{\odot}$, which agrees within a factor of two with the mass estimates from millimeter continuum emission and virial mass from CS.

Once the mass of the complex and of the individual clumps were obtained, we are able to investigate whether the clumps at low velocity (MM1 and MM6) and the complex of clumps at high velocity are bounded. Considering that the velocity difference $v_{\text {dif }}$ of the two systems is $11.9 \mathrm{~km} \mathrm{~s}^{-1}$, and that the projected distance $r_{p}$ from their respective center of mass is $\sim 5 \mathrm{pc}$, a gravitationally bounded system will require a mass of $\sim 2 \times 10^{5} M_{\odot}$, exceeding the total mass of the clumps by a factor of 10 . Then, we discarded that the low velocity clumps are gravitationally bounded to the complex. We cannot rule out for the moment other kind of interaction between these two systems.

\subsubsection{Rotational Temperatures of the G331.512-0.103 Outflow}

When observations of several transitions in a particular molecule are available, it is possible to estimate the rotational temperature, $T_{\text {rot }}$, and the total column density, $N_{T}$, from a rotational diagram analysis, assuming that the lines are optically thin and that excitation temperatures follow $T_{\mathrm{ex}} \gg T_{\mathrm{bg}}$ (e.g., Linke et al. 1979; Blake et al. 1987; Garay et al. 2002). We considered the equation

$$
\frac{N_{k}}{g_{k}}=\frac{3 k \int T_{\mathrm{mb}} d v}{8 \pi^{3} \mu^{2} v S},
$$

with $S$ the intrinsic line strength, $\mu$ the permanent dipole moment, and $N_{k}$ and $g_{k}$ the column density and degeneracy of the upper transition state. If we assume that a single rotational temperature is responsible for the thermalization of the population distribution, then

$$
\frac{N_{k}}{g_{k}}=\frac{N_{T}}{Q\left(T_{\text {rot }}\right)} \exp \left(-E_{u} / k T_{\text {rot }}\right),
$$

with $N_{T}$ is the total molecular column density summed over all the levels, $E_{u}$ is the energy of the upper transition state, and $Q\left(T_{\text {rot }}\right)$ is the rotational partition function at temperature $T_{\text {rot }}$.

The previous analysis was made on the redshifted and blueshifted wing emission of the G331.512-0.103 outflow, using the $\mathrm{SiO}$ transitions $J=7 \rightarrow 6$ and $J=8 \rightarrow 7$, and the SO transitions $J_{K}=8_{8} \rightarrow 7_{7}$ and $J_{K}=8_{7} \rightarrow 7_{6}$. We consider two ranges of velocity for integration: -144.1 to -95.6 , and -79.9 to -16.3 . The values computed for $T_{\text {rot }}, Q\left(T_{\text {rot }}\right)$ and $N_{T}$ from
Table 11

Outflow Parameters Obtained from Rotational Diagrams

\begin{tabular}{lcccc}
\hline \hline Molecule & Velocity Range & $\begin{array}{c}T_{\text {rot }} \\
(\mathrm{K})\end{array}$ & $Q\left(T_{\text {rot }}\right)$ & $\begin{array}{c}N_{T} \\
\left(10^{13} \mathrm{~cm}^{-2}\right)\end{array}$ \\
\hline $\mathrm{SiO}$ & $-144.1 \leftrightarrow-95.6$ & $123.2 \pm 15.2$ & 118.3 & 3.26 \\
& $-79.9 \leftrightarrow-16.3$ & $138.0 \pm 29.4$ & 132.5 & 2.56 \\
\hline $\mathrm{SO}$ & $-144.1 \leftrightarrow-95.6$ & $93.7 \pm 4.8$ & 254.3 & 45.93 \\
& $-79.9 \leftrightarrow-16.3$ & $77.3 \pm 4.4$ & 207.1 & 37.95 \\
\hline
\end{tabular}

the diagrams are tabulated in Table 11 . The uncertainty in the estimates of rotational temperatures are obtained using the error in the estimate of $I\left(T_{\mathrm{MB}}\right)=I\left(T_{A}^{*}\right) / \eta_{\mathrm{MB}}$ (see Equation (1)), and propagating them through Equations (14) and (15).

For $\mathrm{SiO}$ transitions, the rotational temperatures of the blue and red wing are $123 \pm 15 \mathrm{~K}$ and $138 \pm 29 \mathrm{~K}$, respectively, with column densities ranging between $2.6 \times 10^{13}$ and $3.3 \times 10^{13} \mathrm{~cm}^{-2}$. For the SO lines, the rotational temperatures of the blue wing $(94 \pm 5 \mathrm{~K})$ are higher than the temperature of the red wing, $(77 \pm 4 \mathrm{~K})$, with column densities varying between $3.8 \times 10^{14}$ and $4.6 \times 10^{14} \mathrm{~cm}^{-2}$. We note that since our estimation of temperatures has been done with only two transitions for each molecule, these values should be considered as a rough estimation and more transitions should be considered for a more robust estimation of the temperature in the wings of the molecular outflow. ALMA observations recently obtained toward this source (Merello et al. 2013) show several transitions of $\mathrm{SO}_{2}$ that will give better determination of rotation temperatures.

\subsubsection{Comparison with Other Massive Star-forming Regions}

In this section, we compare the characteristics of the clumps in G331.5-0.1 with those of the sample of 42 massive star-forming regions presented by Molinari et al. (2008). In that study, a 8-1200 $\mu \mathrm{m}$ SED for each YSO is presented, using MSX, IRAS, and sub-mm $/ \mathrm{mm}$ data, aiming to relate their envelope mass with the bolometric luminosity. Those sources that were fitted with an embedded ZAMS envelope were named as "IR," and those fitted only with a modified blackbody peaking at large wavelengths were named "MM." A further classification, as a "P" or "S" source, was made according whether or not they were the primary (most massive) object in the field. The parameters fitted for MM sources gave temperatures with a median value of $\sim 20 \mathrm{~K}$, and spectral indexes $\beta$ with median value $\sim 1.5$. Our sample of millimeter clumps in the G331.5-0.1 GMC central region seems to correspond to the MM sources in their sample.

Figure 16 shows a $L_{\mathrm{bol}}-M_{\mathrm{env}}$ diagram for the sample of 42 sources from Molinari et al. (2008), including the values derived for our six millimeter clumps (filled circles). Clearly, our sources are more massive and luminous that most of the objects in that sample, with bolometric luminosities in general an order of magnitude larger than their MM sources. It is worth noting that the six clumps in the G331.5-0.1 GMC central region fall in the $L_{\text {bol }}-M_{\text {env }}$ diagram toward the prolongation of the isochrone of $\sim 10^{5} \mathrm{yr}$ shown in Figure 9 of Molinari et al. (2008). This trend is associated with the end of the accelerated accretion phase, and therefore our sample of millimeter clumps MM1-MM6 may be in the phase of envelope clean-up, observationally identified with Hot Cores and UC H II regions by Molinari et al.

Similar conclusions can be obtained when compared with the study with Herschel of star-forming compact dust sources from Elia et al. (2010), where modified blackbody SED fitting was made for sources extracted in two $2^{\circ} \times 2^{\circ}$ Galactic plane fields 


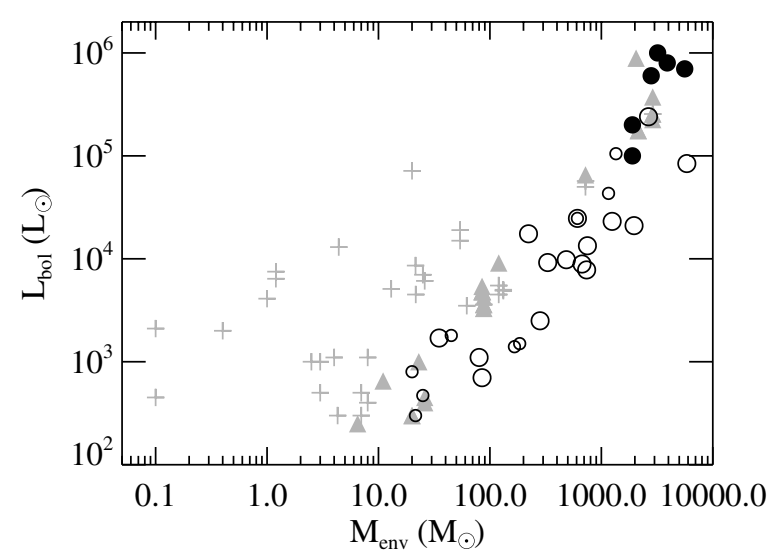

Figure 16. $L_{\mathrm{bol}}-M_{\mathrm{env}}$ diagram for the six millimeter clumps (MM1-MM6) in the G331.5-0.1 GMC central region (filled black circles), together with sources from Molinari et al. (2008). From the Molinari et al. sample, MM sources are represented as open circles (small representing MM-S sources), IR-P sources are shown in gray filled triangles, and IR-S are indicated in crosses.

centered at $l=30^{\circ}, b=0^{\circ}$, and $l=59^{\circ}, b=0^{\circ}$ using the five Hi-GAL bands $(70,160,250,350$, and $500 \mu \mathrm{m})$. Our sample of millimeter clumps remains in the extreme of most luminous and massive millimeter sources.

\section{SUMMARY}

Observations at several wavelengths show that the G331.5$0.1 \mathrm{GMC}$ central region is one of the largest, most massive, and most luminous regions of massive star formation in the Galactic disk. We have obtained a multi-tracer view of this source using radio, (sub)millimeter, and infrared images, starting from an analysis of the global parameters of the parent GMC of this region at a scale of $1^{\circ}$, and continuing to smaller scales until identifying and resolving features at a scale inside G331.5-0.1 of one arcsecond.
Millimeter maps reveal that the G331.5-0.1 GMC central region harbors six massive clumps within a region of $15 \mathrm{pc}$ in diameter. The average size, mass, and molecular hydrogen density of these clumps are $1.6 \mathrm{pc}, 3.2 \times 10^{3} M_{\odot}$, and $3.7 \times$ $10^{4} \mathrm{~cm}^{-3}$, respectively. From the SED analysis of the clumps, we estimated an average dust temperature and bolometric luminosity of $32 \mathrm{~K}$ and $5.7 \times 10^{5} L_{\odot}$, respectively. These values are similar to those of massive and dense clumps typically found in high-mass, star-forming regions. The high number of massive and dense clumps within G331.5-0.1 makes it one of the most densely populated GMC central regions in the Milky Way. At the center of the brightest, most massive, and densest molecular clump within G331.5-0.1 central region, we discovered one of the most luminous and massive protostellar objects presently known, which drives a powerful molecular outflow and a thermal jet. The outflow is not resolved at a resolution of $\sim 7^{\prime \prime}$, so it could be directed along the line of sight. Further high resolution observations with ALMA are underway and will be reported elsewhere.

We found four compact radio sources along the G331.5-0.1 central region. Two of them have a spectral index consistent with ionized stellar winds, which originated from young luminous objects. In particular, one of these radio sources is located at the center of the molecular outflow clump, which suggests that is associated with its driving source.

L.B. and G.G. gratefully acknowledge support from CONICYT through projects FONDAP 15010003 and BASAL PFB06. M.M. was supported by a Fulbright Fellowship and the NSF grant AST-1109116. We thank our referee, M. Pestalozzi, for very useful comments that improved the clarity of this paper. We would like to dedicate this paper to the memory of our friend and collaborator Jorge May Humeres (1936-2011), whose inspiration and advice in many ways made this work possible. He is missed deeply.

\section{APPENDIX}

Figures 17-20 present maps of the observed spectra.

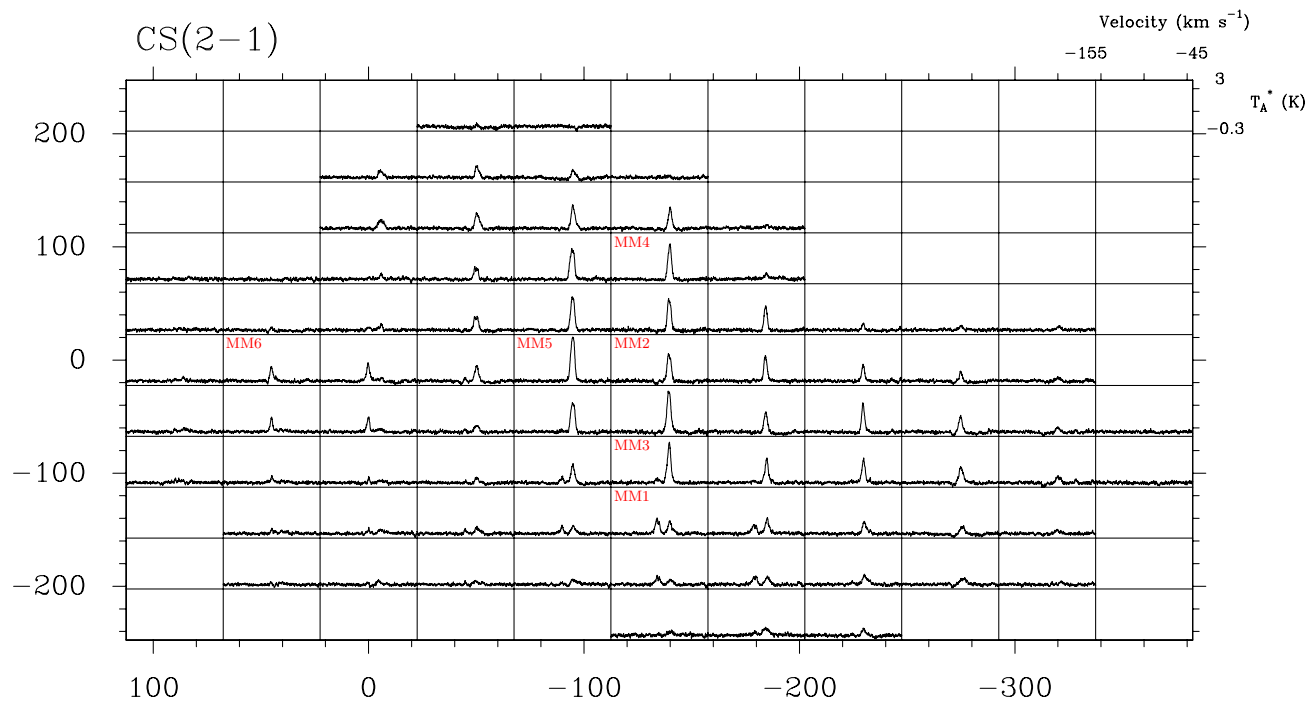

Figure 17. Observed spectra of the $\operatorname{CS}(2 \rightarrow 1)$ line emissions toward the G331.5-0.1 GMC central region. The grid spacing is $45^{\prime \prime}$. Offsets are from the reference position at $\alpha_{2000}=16^{\mathrm{h}} 12^{\mathrm{m}} 24.5$ and $\delta_{2000}=-51^{\circ} 27^{\prime} 29^{\prime \prime} \cdot 98$. In each box the velocity scale ranges from -155 to $-45 \mathrm{~km} \mathrm{~s}^{-1}$. The antenna temperature scale is from -0.3 to $3 \mathrm{~K}$. The labels MM1 to MM6 show the positions in the grid closer to the peak position of the millimeter clumps, defined in Section 3.2.1.

(A color version of this figure is available in the online journal.) 


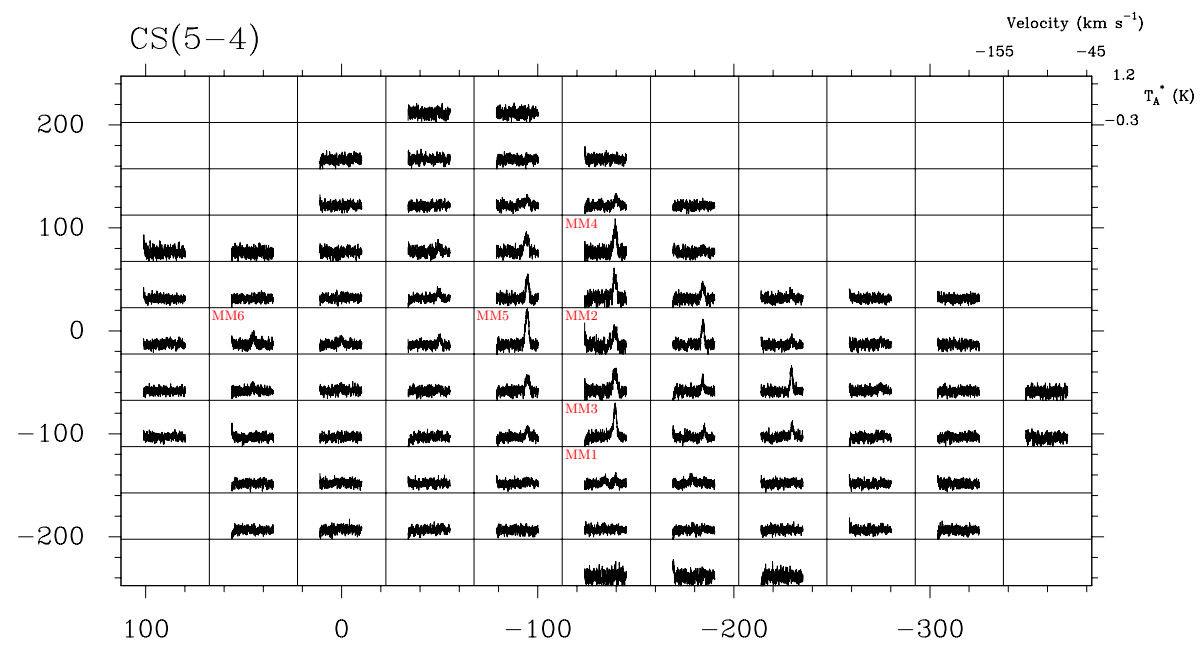

Figure 18. Observed spectra of the CS(5 $\rightarrow 4)$ line emissions toward the G331.5-0.1 GMC central region. The grid spacing is $45^{\prime \prime}$. Offsets are from the reference position at $\alpha_{2000}=16^{\mathrm{h}} 12^{\mathrm{m}} 24^{\mathrm{s}} .5$ and $\delta_{2000}=-51^{\circ} 27^{\prime} 29^{\prime \prime} \cdot 98$. In each box the velocity scale ranges from -155 to $-45 \mathrm{~km} \mathrm{~s}^{-1}$. The antenna temperature scale is from -0.3 to $1.2 \mathrm{~K}$. The labels MM1 to MM6 show the positions in the grid closer to the peak position of the millimeter clumps, defined in Section 3.2.1.

(A color version of this figure is available in the online journal.)

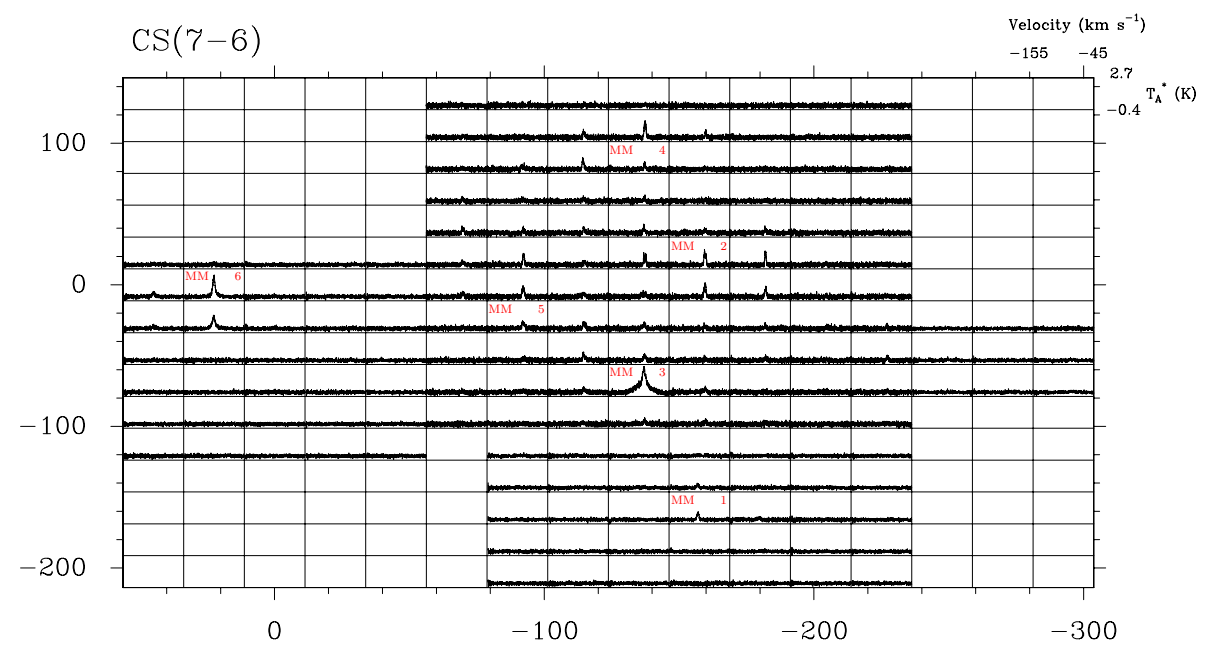

Figure 19. Observed spectra of the $\mathrm{CS}(7 \rightarrow 6)$ line emissions toward the G331.5-0.1 central region. The grid spacing is 22 ". 5 . Offsets are from the reference position at $\alpha_{2000}=16^{\mathrm{h}} 12^{\mathrm{m}} 24.5$ and $\delta_{2000}=-51^{\circ} 27^{\prime} 29^{\prime \prime}$. . In each box the velocity scale ranges from -155 to $-45 \mathrm{~km} \mathrm{~s}^{-1}$. The antenna temperature scale is from -0.4 to 2.7 K. The labels MM1 to MM6 show the positions in the grid closer to the peak position of the millimeter clumps, defined in Section 3.2.1.

(A color version of this figure is available in the online journal.)

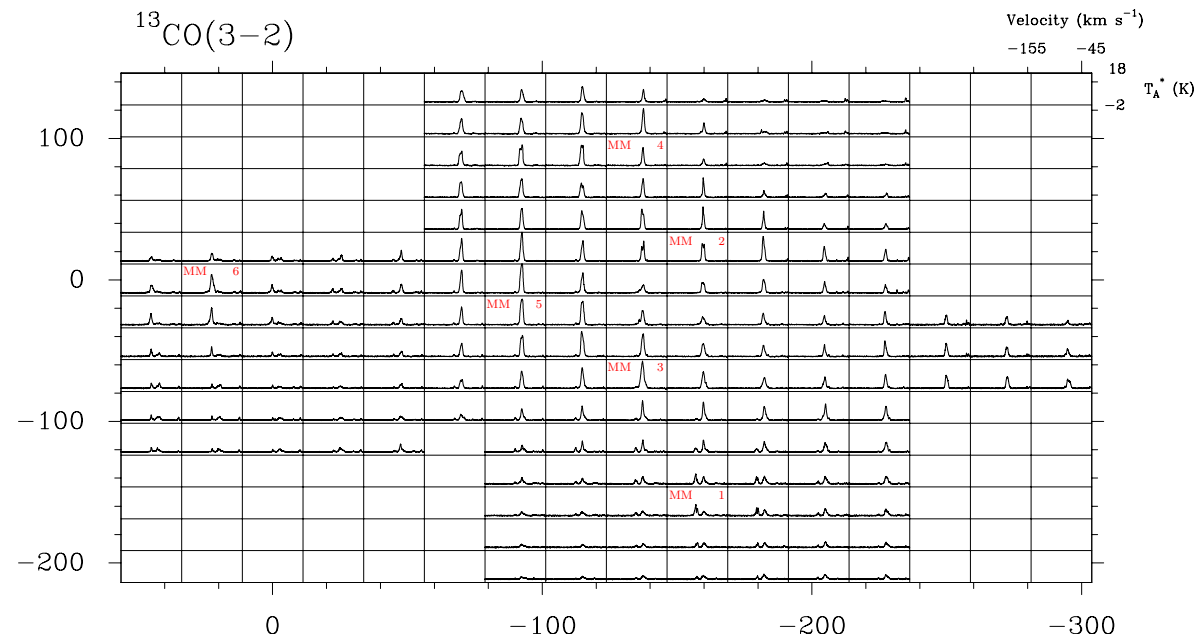

Figure 20. Observed spectra of the ${ }^{13} \mathrm{CO}(3 \rightarrow 2)$ line emissions toward the G331.5-0.1 central region. The grid spacing is 22 ". 5 . Offsets are from the reference position at $\alpha_{2000}=16^{\mathrm{h}} 12^{\mathrm{m}} 24^{\mathrm{s}} .5$ and $\delta_{2000}=-51^{\circ} 27^{\prime} 29^{\prime \prime}$. 9 . In each box the velocity scale ranges from -155 to $-45 \mathrm{~km} \mathrm{~s}^{-1}$. The antenna temperature scale is from -2 to $18 \mathrm{~K}$. The labels MM1 to MM6 show the positions in the grid closer to the peak position of the millimeter clumps, defined in Section 3.2.1.

(A color version of this figure is available in the online journal.) 


\section{REFERENCES}

Alvarez, H., May, J., \& Bronfman, L. 1990, ApJ, 348, 495

Bally, J., Moeckel, N., \& Throop, H. 2005, in ASP Conf. Ser. 341, Chondrites and the Protoplanetary Disk, ed. A. N. Krot, E. R. D. Scott, \& B. Reipurth (San Francisco, CA: ASP), 81

Bartkiewicz, A., \& van Langevelde, H. J. 2012, in IAU Symp. 287, Cosmic Masers-from OH to H0, ed. R. S. Booth, E. M. L. Humphreys, \& W. H. T. Vlemmings (Cambridge: Cambridge Univ. Press), 117

Blake, G. A., Sutton, E. C., Masson, C. R., \& Phillips, T. G. 1987, ApJ, 315,621

Bourke, T. L., Garay, G., Lehtinen, K. K., et al. 1997, ApJ, 476, 781

Bronfman, L., Alvarez, H., Cohen, R. S., \& Thaddeus, P. 1989, ApJS, 71,481

Bronfman, L., Casassus, S., May, J., \& Nyman, L.-Å. 2000, A\&A, 358,521

Bronfman, L., Cohen, R. S., Thaddeus, P., \& Alvarez, H. 1985, in IAU Symp. 106, The Milky Way Galaxy, ed. H. van Woerden, R. J. Allen, \& W. B. Burton (Dordrecht: Reidel), 331

Bronfman, L., Garay, G., Merello, M., et al. 2008, ApJ, 672, 391

Bronfman, L., Nyman, L.-A., \& May, J. 1996, A\&AS, 115, 81

Caswell, J. L. 1997, MNRAS, 289, 203

Caswell, J. L. 1998, MNRAS, 297, 215

Caswell, J. L. 2009, PASA, 26, 454

Caswell, J. L., Fuller, G. A., Green, J. A., et al. 2011, MNRAS, 417, 1964

Caswell, J. L., \& Haynes, R. F. 1987, A\&A, 171, 261

Caswell, J. L., Haynes, R. F., \& Goss, W. M. 1980, AuJPh, 33, 639

Caswell, J. L., Vaile, R. A., Ellingsen, S. P., Whiteoak, J. B., \& Norris, R. P. 1995, MNRAS, 272, 96

Cesaroni, R., Walmsley, C. M., Koempe, C., \& Churchwell, E. 1991, A\&A, 252,278

Churchwell, E., Walmsley, C. M., \& Cesaroni, R. 1990, A\&AS, 83, 119

Dunham, M. K., Rosolowsky, E., Evans, N. J., II, Cyganowski, C., \& Urquhart, J. S. 2011, ApJ, 741, 110

Edgar, R., \& Clarke, C. 2004, MNRAS, 349, 678

Elia, D., Schisano, E., Molinari, S., et al. 2010, A\&A, 518, L97

Faúndez, S., Bronfman, L., Garay, G., et al. 2004, A\&A, 426, 97

Fazio, G. G., Hora, J. L., Allen, L. E., et al. 2004, ApJS, 154, 10

Fukui, Y., Ogawa, H., Kawabata, K., Mizuno, A., \& Sugitani, K. 1991, in IAU Symp. 148, The Magellanic Clouds, ed. R. Haynes \& D. Milne (Dordrecht: Kluwer), 105

Fukui, Y., \& Sakakibara, O. 1992, Mitsubishi Electronic Advance, 60, 11

Furuya, R. S. 2003, in ASP Conf. Ser. 287, Galactic Star Formation Across the Stellar Mass Spectrum, ed. J. M. De Buizer \& N. S. van der Bliek (San Francisco, CA: ASP), 367

Garay, G., Brooks, K. J., Mardones, D., Norris, R. P., \& Burton, M. G. 2002, ApJ, 579,678

Garay, G., \& Lizano, S. 1999, PASP, 111, 1049

Goss, W. M., Manchester, R. N., \& Robinson, B. J. 1970, AuJPh, 23, 559

Goss, W. M., Radhakrishnan, V., Brooks, J. W., \& Murray, J. D. 1972, ApJS, 24, 123

Gottlieb, C. A., Gottlieb, E. W., Litvak, M. M., Ball, J. A., \& Penfield, H. 1978, ApJ, 219, 77

Heiderman, A., Evans, N. J., II, Allen, L. E., Huard, T., \& Heyer, M. 2010, ApJ, 723, 1019

Jones, C., \& Dickey, J. M. 2012, ApJ, 753, 62

Kahn, F. D. 1974, A\&A, 37, 149

Kennicutt, R. C., Jr. 1998, ARA\&A, 36, 189
Kennicutt, R. C. 2005, in IAU Symp. 227, Massive Star Birth: A Crossroads of Astrophysics, ed. R. Cesaroni, M. Felli, E. Churchwell, \& M. Walmsley (Cambridge: Cambridge Univ. Press), 3

Kennicutt, R. C., \& Evans, N. J. 2012, ARA\&A, 50, 531

Kerr, F. J., \& Knapp, G. R. 1970, AuJPA, 18, 9

Krumholz, M. R., \& McKee, C. F. 2008, Natur, 451, 1082

Kuiper, R., Klahr, H., Beuther, H., \& Henning, T. 2010, ApJ, 722, 1556

Lada, C. J., Lombardi, M., \& Alves, J. F. 2010, ApJ, 724, 687

Larson, R. B., \& Starrfield, S. 1971, A\&A, 13, 190

Linke, R. A., Frerking, M. A., \& Thaddeus, P. 1979, ApJL, 234, L139

Martin-Pintado, J., Bachiller, R., \& Fuente, A. 1992, A\&A, 254, 315

McKee, C. F., \& Ostriker, E. C. 2007, ARA\&A, 45, 565

Menten, K. M., Walmsley, C. M., Henkel, C., et al. 1986, A\&A, 169, 271

Merello, M., Bronfman, L., Garay, G., et al. 2013, ApJL, in press (arXiv:1307.6244)

Miettinen, O., Harju, J., Haikala, L. K., \& Pomrén, C. 2006, A\&A, 460, 721

Mill, J. D., O’Neil, R. R., Price, S., et al. 1994, JSpRo, 31, 900

Molinari, S., Pezzuto, S., Cesaroni, R., et al. 2008, A\&A, 481, 345

Myers, P. C. 1995, in Molecular Clouds and Star Formation, ed. C. Yuan \& J.-H. You (Singapore: World Scientific), 47

Neugebauer, G., Habing, H. J., van Duinen, R., et al. 1984, ApJL, 278, L1

Ogawa, H., Mizuno, A., Ishikawa, H., Fukui, Y., \& Hoko, H. 1990, IJIMW, 11,717

Ossenkopf, V., \& Henning, T. 1994, A\&A, 291, 943

Panagia, N. 1973, AJ, 78, 929

Pestalozzi, M. R., Minier, V., \& Booth, R. S. 2005, A\&A, 432, 737

Plambeck, R. L., Wright, M. C. H., Welch, W. J., et al. 1982, ApJ, 259, 617

Plume, R., Jaffe, D. T., \& Evans, N. J., II. 1992, ApJS, 78, 505

Reynolds, S. P. 1986, ApJ, 304, 713

Rydbeck, O. E. H., Hjalmarson, A., Rydbeck, G., et al. 1980, ApJL, 235, L171

Sault, R. J., Teuben, P. J., \& Wright, M. C. H. 1995, in ASP Conf. Ser. 77, Astronomical Data Analysis Software and Systems IV, ed. R. A. Shaw, H. E. Payne, \& J. J. E. Hayes (San Francisco, CA: ASP), 433

Schilke, P., Walmsley, C. M., Pineau des Forets, G., \& Flower, D. R. 1997, A\&A, 321, 293

Schuller, F., Menten, K. M., Contreras, Y., et al. 2009, A\&A, 504, 415

Shaver, P. A., \& Goss, W. M. 1970, AuJPA, 14, 133

Shirley, Y. L., Evans, N. J., II, Young, K. E., Knez, C., \& Jaffe, D. T. 2003, ApJS, 149,375

Swade, D. A. 1989, ApJ, 345, 828

Szymczak, M., Kus, A. J., Hrynek, G., Kěpa, A., \& Pazderski, E. 2002, A\&A, 392,277

Urquhart, J. S., Hoare, M. G., Lumsden, S. L., Oudmaijer, R. D., \& Moore, T. J. T. 2008, in ASP Conf. Ser. 387, Massive Star Formation: Observations Confront Theory, ed. H. Beuther, H. Linz, \& T. Henning (San Francisco, CA: ASP), 381

Welch, W. J., Wright, M. C. H., Plambeck, R. L., Bieging, J. H., \& Baud, B. 1981, ApJL, 245, L87

Williams, J. P., Blitz, L., \& McKee, C. F. 2000, in Protostars and Planets IV, ed. V. Mannings, A. P. Boss, \& S. S. Russell (Tucson, AZ: Univ. Arizona Press), 97

Wilson, T. L., Rohlfs, K., \& Hüttemeister, S. (ed.) 2009, Tools of Radio Astronomy (Berlin: Springer)

Wilson, T. L., \& Rood, R. 1994, ARA\&A, 32, 191

Wolfire, M. G., \& Cassinelli, J. P. 1987, ApJ, 319, 850

Yorke, H. W., \& Kruegel, E. 1977, A\&A, 54, 183

Zinnecker, H., \& Yorke, H. W. 2007, ARA\&A, 45, 481 